\title{
VLT spectropolarimetry of the fast expanding type la SN 2006X $X^{\star} \star \star$
}

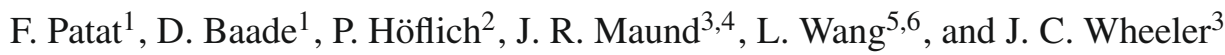 \\ 1 European Organization for Astronomical Research in the Southern Hemisphere (ESO), K. Schwarzschild-str. 2, \\ 85748 Garching b. München, Germany \\ e-mail: fpatat@eso.org \\ 2 Department of Physics, Florida State University, Tallahassee, Florida 32306-4350, USA \\ 3 Department of Astronomy and McDonald Observatory, The University of Texas at Austin, Austin, TX 78712, USA \\ 4 Department of Physics, Texas A\&M University, College Station, Texas 77843, USA \\ 5 Dark Cosmology Centre, Niels Bohr Institute, University of Copenhagen, Juliane Maries Vej 30, 2100 Copenhagen, Denmark \\ ${ }^{6}$ Purple Mountain Observatory, 2 West Bejing Road, Nanjing 210008, China \\ Received 22 July 2008 / Accepted 15 September 2009
}

\section{ABSTRACT}

\begin{abstract}
Aims. The main goal of this study is to probe the ejecta geometry and to get otherwise unobtainable information about the explosion mechanism of type Ia Supernovae (SNe).

Methods. Using VLT-FORS1 we performed optical spectropolarimetric observations of the type Ia SN 2006X on 7 pre-maximum epochs (day -10 to day -1 ) and one post-maximum epoch (+39 days).

Results. The SN shows strong continuum interstellar polarization reaching about $8 \%$ at $4000 \AA$, characterized by a wavelength dependency that is substantially different from that of the Milky-Way dust mixture. Several SN features, like Si II $6355 \AA$ and the Ca II IR triplet, present a marked evolution. The Ca II near-IR triplet shows a pronounced polarization $(\sim 1.4 \%)$ already on day -10 in correspondence with a strong high-velocity feature (HVF). The Si II polarization peaks on day -6 at about $1.1 \%$ and decreases to $0.8 \%$ on day -1 . By day +39 no polarization signal is detected for the Si II line, while the Ca II IR triplet shows a marked repolarization at the level of $1.2 \%$. As in the case of another strongly polarized SN (2004dt), no polarization was detected across the O I $7774 \AA$ A absorption.

Conclusions. The fast-expanding SN 2006X lies on the upper edge of the relation between peak polarization and decline rate, and it confirms previous speculations about a correlation between degree of polarization, expansion velocity, and HVF strength. The polarization of $\mathrm{Ca}$ II detected in our last epoch, the most advanced ever obtained for a type Ia SN, coincides in velocity with the outer boundary of the Ca synthesized during the explosion (15000-17000 km s$\left.{ }^{-1}\right)$ in delayed-detonation models. This suggests a large scale chemical inhomogeneity as produced by off-center detonations, a rather small amount of mixing, or a combination of both effects. In contrast, the absence of polarization at the inner edge of the Ca-rich layer (8000-10 $\left.000 \mathrm{~km} \mathrm{~s}^{-1}\right)$ implies a substantial amount of mixing in these deeper regions.
\end{abstract}

Key words. stars: supernovae: general - stars: supernovae: individual: 2006X - ISM: general - dust, extinction

\section{Introduction}

The importance of type Ia Supernovae (hereafter SNe Ia) cannot be overstated. Besides their fundamental role in the chemical enrichment of galaxies, in the past ten years they have played a crucial part in Cosmology, bringing new and exciting insights into the history of the Universe and its future evolution (Riess et al. 1998; Perlmutter et al. 1998). For these reasons, SNe Ia are being studied by several extended surveys, which aim at pinning down the cosmological parameters. Notwithstanding these extensive efforts, the understanding of fundamental issues like the progenitor's nature, the explosion mechanisms, and empirical relations such as luminosity vs. light curve shape, necessarily rely on the study of the nearby objects.

During the last decade, especially after the advent of $8 \mathrm{~m}$ class telescopes, a new aspect of the SN phenomenon has come within the reach of investigation, i.e. the geometry of the explosion. Since the electron scattering in asymmetric SN ejecta is

\footnotetext{
^ Based on observations made with ESO Telescopes at Paranal Observatory under Program IDs 076.D-0177 and 076.D-0178.

$\star \star$ Appendix A is only available in electronic form at http://www . aanda. org
}

expected to produce a net linear polarization (see for instance Höflich 1991), the ejecta geometry and clumping can be directly probed by means of polarimetry. Starting with the first broadband studies (Wang et al. 1996a), two groups have been collecting unique information through the spectropolarimetric study of a number of SNe Ia (Wang et al. 1997, 2001; Howell et al. 2001; Kasen et al. 2003; Wang et al. 2003; Hoeflich et al. 2006; Leonard et al. 2005; Wang et al. 2006b; Chornock \& Filippenko 2007) that have led to important insights on explosion asymmetry and ejecta clumping that no other technique can provide (Leonard et al. 2005; Wang et al. 2007; Wang \& Wheeler 2008).

Here we present low-resolution optical spectropolarimetry of SN 2006X obtained at the ESO Very Large Telescope, spanning from 10 days before $B$ maximum light to about 40 days past maximum. To the best of our knowledge, in terms of evolution coverage, this is the most complete spectropolarimetric data set ever obtained for a type Ia SN, equaled only by SN 2001el (Wang et al. 2003).

SN 2006X was discovered by Suzuki \& Migliardi (2006) on 4 February 2006 (UT) in the Virgo Cluster spiral galaxy NGC 4321 (see Fig. 1), located at a distance of $16.1 \pm$ 1.3 Mpc (Ferrarese et al. 1996) and receding with a velocity $v_{\text {gal }}=1572 \mathrm{~km} \mathrm{~s}^{-1}$ (Rand 1995). A few days later, the object 


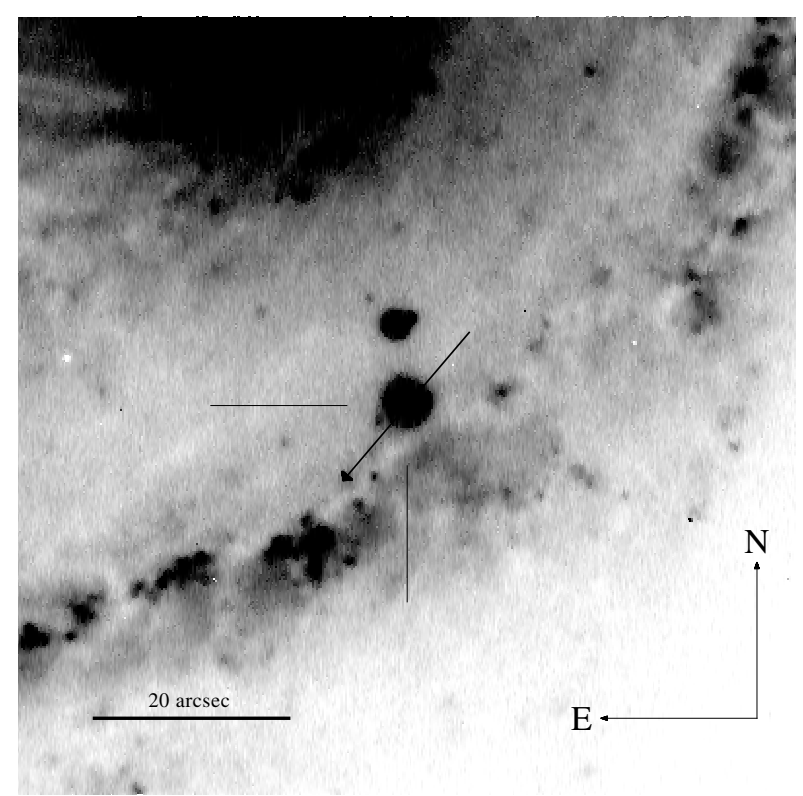

Fig. 1. SN 2006X in NGC 4321. The $R$ band image was obtained with VLT-FORS1 (see Sect. 2) on February 11, 2006 (30 s exposure time). The arrow placed at the SN position indicates the inter-stellar polarization position angle (139.5 degrees; see Sect. 4).

was classified as a normal type Ia event 1-2 weeks before maximum light and most likely affected by a substantial extinction (Quimby et al. 2006a). Prompt VLA observations have shown no radio source at the SN position (Stockdale et al. 2006), actually establishing one of the deepest and earliest limits for radio emission from a type Ia, implying a mass-loss rate of less than a few $10^{-8} M_{\odot} \mathrm{yr}^{-1}$, for a wind velocity of $10 \mathrm{~km} \mathrm{~s}^{-1}$ and assuming a spherically symmetric, steady wind. Observations carried out with XRT on board Swift put an upper limit of $1.5 \times 10^{39} \mathrm{erg} \mathrm{s}^{-1}$ for the X-ray flux in the $0.2-10 \mathrm{keV}$ band (Immler et al. 2006), corresponding to a mass loss rate lower than $7 \times 10^{-6} M_{\odot} \mathrm{yr}^{-1}$. The SN was also reported to show a rather strong continuum linear polarization in the optical domain, reaching 8 per cent in the blue and decreasing to 3.5 per cent in the red, with a wavelength dependency significantly different from that typical of extinguished stars in our own galaxy (Wang et al. 2006a). High resolution spectroscopy around maximum light has shown very intense interstellar absorption features, identified as $\mathrm{CN}, \mathrm{Na} \mathrm{I}$, $\mathrm{Ca}$ II and K I, confirming the presence of significant extinction along the line of sight (Lauroesch et al. 2006; Patat et al. 2007; Cox \& Patat 2008). Rather remarkably, the multi-epoch high resolution spectroscopy has also revealed a pronounced evolution in a number of the Na ID components, which have been interpreted as signatures of circum-stellar material lost by the progenitor system before the explosion (Patat et al. 2007, but see also Chugai 2008). An extensive optical and near-infrared study has been presented by Wang et al. (2008a). They have shown that this SN has one of the highest expansion velocities ever observed, and this is accompanied by slightly peculiar light and color curves. These might be explained by a light echo and/or the interaction between the SN ejecta and the circumstellar material (see also Wang et al. 2008b; Crotts \& Yourdon 2008). The reddening corrected value for the decline rate of SN 2006X is $\Delta m_{15}(B)=1.31 \pm 0.05$. The normalized $\left(\Delta m_{15}=1.1\right)$ absolute $V$ magnitude is $-19.2 \pm 0.2$, which is consistent with those of fiducial type Ia SNe, while it is $0.2-0.3 \mathrm{mag}$ fainter in $I, J$ and $K$ bands (Wang et al. 2008a).
In this paper we report the results of a spectropolarimetric campaign conducted with VLT-FORS1, covering seven premaximum phases from day -10 to day -1 . The data set includes an additional epoch 39 days past maximum light. The paper is organized as follows. In Sect. 2 we present and discuss our observations and data reduction. The spectroscopic evolution during the phases covered by our observations is analyzed in Sect. 3. Section 4 deals with inter-stellar polarization, while Sect. 5 presents the SN intrinsic polarization. Finally, in Sect. 6 we discuss our results and summarize our conclusions. Additional figures are presented in Appendix A, available online.

\section{Observations and data reduction}

We observed SN 2006X on 9 different epochs, using the FOcal Reducer/low-dispersion Spectrograph (hereafter FORS1), mounted at the Cassegrain focus of the ESO-Kueyen $8.2 \mathrm{~m}$ telescope (Appenzeller et al. 1998). In this multi-mode instrument, equipped with a $2048 \times 2048$ pixel (px) TK2048EB4-1 backside-thinned CCD, polarimetry is performed introducing a Wollaston prism (19" throw) and a super-achromatic half-wave plate. In order to reduce some known instrumental problems (see Patat \& Romaniello 2006) we always used 4 half-wave plate (HWP) angles (0, 22.5, 45 and 67.5 degrees). Exposure times ranged from 5 to 15 minutes per plate angle and, in some cases, the sequence was repeated in order to increase the signal-tonoise ratio. All spectra were obtained with the low-resolution G300V grism coupled to a 1.1 arcsec slit, giving a spectral range 3300-8600 $\AA$, a dispersion of $\sim 2.9 \AA$ pixel $^{-1}$ and a resolution of $12.4 \AA(F W H M)$ at $5800 \AA$. Data were bias, flat-field corrected and wavelength calibrated by means of standard tasks within IRAF $^{1}$. The rms error on the wavelength calibration is about $0.7 \AA$. The ordinary (upper) and extraordinary (lower) beams were processed separately. Stokes parameters, linear polarization degree and position angle were computed by means of specific routines written by us. Finally, polarization bias correction and error estimates were performed following the prescriptions described by Patat \& Romaniello (2006), while the HWP zeropoint angle chromatism was corrected using the tabulated data (Jehin et al. 2002). In order to increase the signal-to-noise ratio, multiple data sets obtained at the same epoch were combined and the final Stokes parameters binned in $\sim 26 \AA$ wide bins (10 pixels). The last two epochs (March 30 and 31) were combined into a single epoch $(+39.2)$.

Flux calibration was achieved through the observation of spectrophotometric standard stars with the full polarimetric optics inserted (HWP angle set to 0 ). Instrumental polarization and position angle offset were checked by observing polarized and unpolarized standard stars, obtained within the FORS1 calibration plan. Finally, the wavelength scale was corrected to the restframe using the host galaxy recession velocity $\left(1572 \mathrm{~km} \mathrm{~s}^{-1}\right.$, Rand 1995).

Because of the rather heavy extinction suffered by SN 2006X (Quimby et al. 2006a; Lauroesch et al. 2006; Wang et al. 2008a; Elias-Rosa et al. 2009, in prep.), the signal-to-noise ratio shortwards of $3900 \AA$ is quite poor. As a consequence, the polarization is not reliably measurable at those short wavelengths. This prevents us from studying the behavior of the Ca II H\&K lines that fall in this region.

\footnotetext{
${ }^{1}$ IRAF is distributed by the National Optical Astronomy Observatories, which are operated by the Association of Universities for Research in Astronomy, under contract with the National Science Foundation.
} 
Table 1. Log of VLT-FORS1 spectropolarimetric observations of SN 2006X.

\begin{tabular}{cccccc}
\hline \hline $\begin{array}{c}\text { Date } \\
\text { (UT) }\end{array}$ & $\begin{array}{c}\text { MJD } \\
(\text { JD-2 400000.5) }\end{array}$ & $\begin{array}{c}\text { Phase } \\
\text { (days) }\end{array}$ & $\begin{array}{c}V \\
(*)\end{array}$ & $\begin{array}{c}\text { airmass } \\
\text { (average) }\end{array}$ & $\begin{array}{c}\text { Exp. time } \\
(\mathrm{s})\end{array}$ \\
\hline $2006-02-09$ & 53775.37 & -10.1 & 15.3 & 1.36 & $4 \times 300$ \\
$2006-02-11$ & 53777.29 & -8.2 & 14.9 & 1.41 & $2 \times 4 \times 300$ \\
$2006-02-12$ & 53778.29 & -7.2 & 14.7 & 1.50 & $2 \times 4 \times 600$ \\
$2006-02-13$ & 53779.22 & -6.3 & 14.5 & 1.68 & $4 \times 600$ \\
$2006-02-16$ & 53782.32 & -3.2 & 14.3 & 1.43 & $4 \times 600$ \\
& & & & & $4 \times 420$ \\
$2006-02-17$ & 53783.31 & -2.2 & 14.2 & 1.39 & $2 \times 4 \times 600$ \\
$2006-02-18$ & 53784.39 & -1.1 & 14.2 & 1.57 & $4 \times 480$ \\
$2006-03-30$ & 53824.20 & +38.7 & 15.9 & 1.34 & $2 \times 4 \times 900$ \\
$2006-03-31$ & 53825.20 & +39.7 & 15.9 & 1.34 & $2 \times 4 \times 900$ \\
\hline
\end{tabular}

(*) $V$ magnitudes are derived from Wang et al. (2008a).

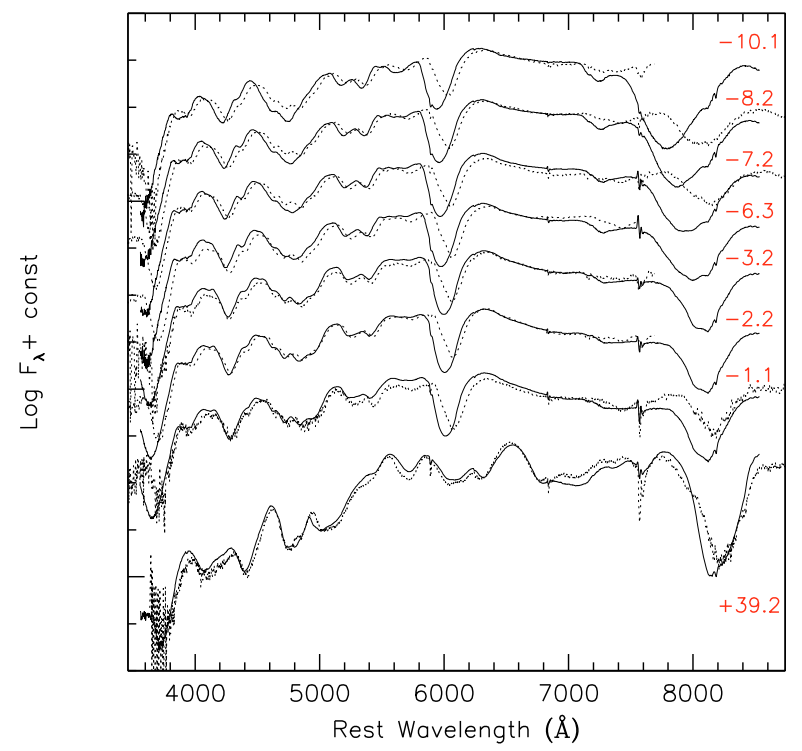

Fig. 2. Spectral evolution of SN 2006X. Phases refer to $B$ maximum light (JD=2 453 786.17; Wang et al. 2008a). For comparison, the spectra of SN 2002bo (Benetti et al. 2004) are also plotted (dotted lines). For presentation, the observed spectra of SN 2002bo (not corrected for extinction) were reddened using a standard extinction law $\left(R_{V}=3.1\right)$ and $E_{B-V}=0.8$.

The log of observations is reported in Table 1, where the $\mathrm{SN}$ phases were computed with respect to the $B$ maximum light ( $\mathrm{JD}=2453$ 786.17, Wang et al. 2008a).

\section{Spectroscopic evolution}

The maximum/post-maximum spectroscopic evolution of SN 2006X has been presented by Wang et al. (2008a), to which we refer the reader for a detailed discussion. Their phase coverage extends up to about 300 days after maximum light and hence our data set is complementary, in the sense that ours mostly covers the pre-maximum epochs. The spectroscopic evolution during these early phases is presented in Fig. 2 where SN 2006X $\left(\Delta m_{15}(B)=1.31 \pm 0.05, M_{V}=-19.2 \pm 0.2\right.$; Wang et al. 2008a) is compared to SN 2002bo $\left(\Delta m_{15}(B)=1.13 \pm 0.05\right.$, $M_{V}=-19.42 \pm 0.33$; Benetti et al. 2004), which was also significantly polarized (Wang et al. 2007, see also Fig. 26 here). The two objects are indeed similar (see Wang et al. 2008a, for a thorough comparison), even though SN 2006X shows definitely higher expansion velocities, especially at early epochs. This

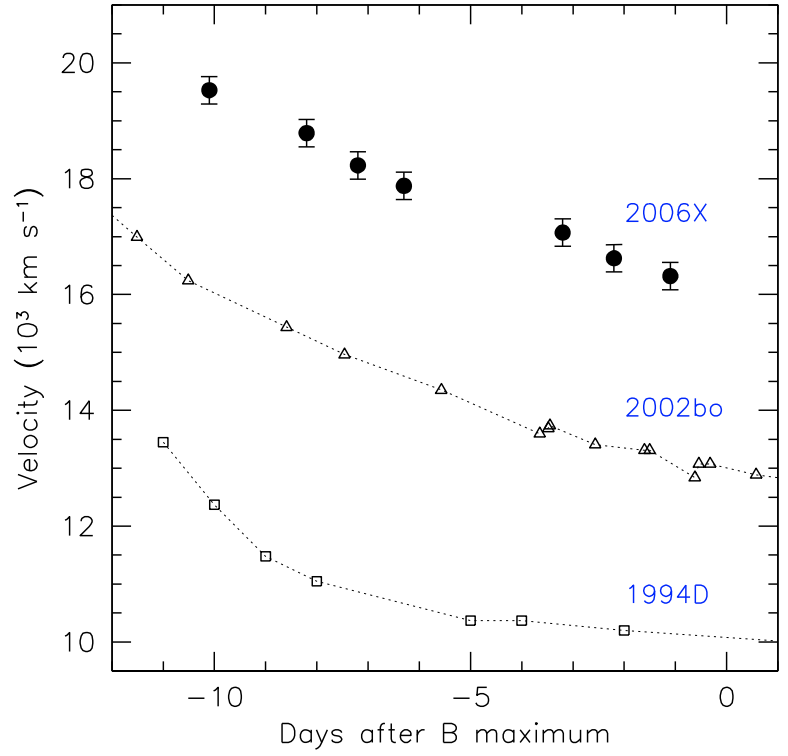

Fig. 3. Evolution of the expansion velocity deduced from the minima of the Si II $6355 \AA$ absorption. For comparison, the data of SN1994D (Patat et al. 1996) and SN 2002bo (Benetti et al. 2004) are also shown.

difference becomes milder and milder as time goes by and at about 40 days past maximum light the two objects are almost indistinguishable. At later epochs the largest discrepancy is seen in the near-IR Ca II triplet absorption trough which, in SN 2006X, extends much further into the blue (see the discussion below and Fig. 4). The evolution of the expansion velocities deduced from the minima of the Si II $6355 \AA$ absorption is presented in Fig. 3 where, for comparison, we have included the data of two other normal SNIa: 1994D (Patat et al. 1996) and 2002bo (Benetti et al. 2004).

As already pointed out by Wang et al. (2008a), the expansion velocities deduced for SN 2006X are exceptionally high (see their Fig. 16 and Fig. 3 here), reaching $\sim 19500 \mathrm{~km} \mathrm{~s}^{-1} 10$ days before $B$ maximum light, i.e. about $3500 \mathrm{~km} \mathrm{~s}^{-1}$ higher than in SN 2002 bo which, from this point of view, was already judged as an unusual event (Benetti et al. 2004, 2005). SN 2006X shows expansion velocities which are even higher than in the extreme case of SN 1984A (Benetti 1989), placing SN 2006X at the upper end of the observed velocity range. During the phases covered by our observations, the velocity decrease is very smooth $\left(\dot{v} \simeq-350 \mathrm{~km} \mathrm{~s}^{-1} \mathrm{day}^{-1}\right)$, as in the case of SN 2002bo, at variance with what is seen in objects like SN 1994D (see Fig. 3) that show significantly lower velocities and a break around 5 days before maximum light (Patat et al. 1996; Benetti et al. 2004). Clearly, as concluded by Wang et al. (2008a), SN 2006X belongs to the High Velocity Gradient (HVG) class proposed by Benetti et al. (2005), that roughly coincides with the broad-line class of Branch et al. (2006).

The value of the $\mathcal{R}(\mathrm{SiII})$ parameter (Nugent et al. 1995) for SN 2006X, estimated from the day -1 spectrum, is $\mathcal{R}(\mathrm{SiII})=0.07 \pm 0.04$, which is exceptionally low if compared to other HVG objects like SN 2002bo $(0.17 \pm 0.05$, Benetti et al. 2004) at maximum light. For comparison, Wang et al. (2008a) report $\mathcal{R}(\mathrm{SiII})=0.12 \pm 0.06$. A similarly low value, $0.05 \pm 0.02$, is obtained for the equivalent width ratio of the two Si II lines, as in Hachinger et al. (2006). Nevertheless, given the fact that the features in SN 2006X are very broad, it is not clear whether this low value is really intrinsic or is rather due to the blending 


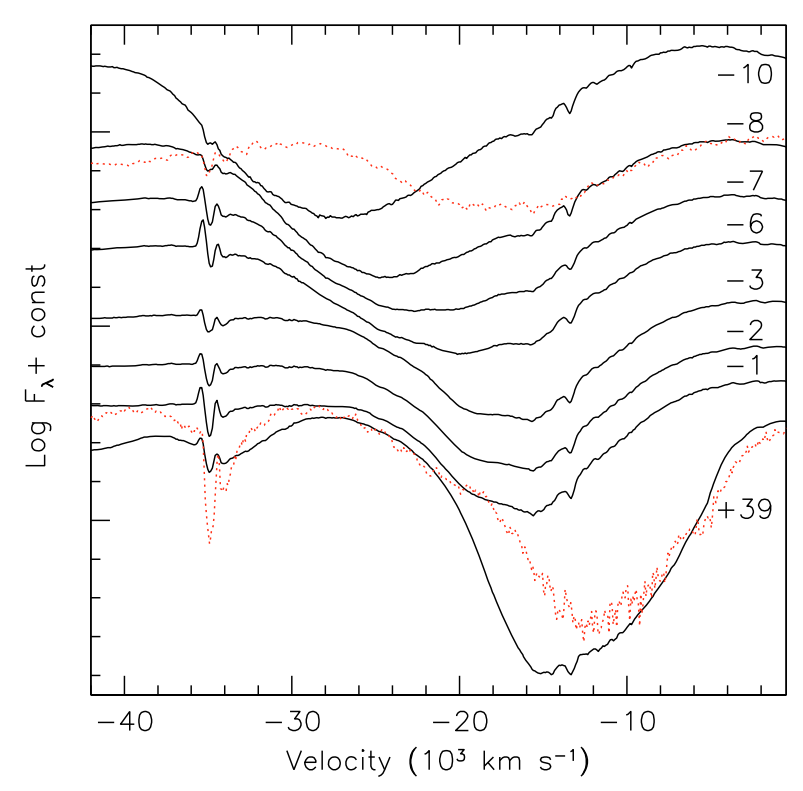

Fig. 4. Evolution of the Ca II IR triplet velocity profile. Velocities are relative to the $g f$-values weighted average triplet wavelength (8567 $\mathrm{A}$ ). The dotted, light-colored curves are the spectra of SN 2002bo (Benetti et al. 2004) at 8.5 days before maximum (top) and 39.9 days after maximum light (bottom).

of the red wing of the Si II $5972 \AA$ A line with the blue wing of the Si II $6355 \AA$ A line (see also Fig. 2).

Another interesting aspect shown by SN 2006X is the presence of a high velocity feature (hereafter HVF), clearly visible in the Ca II IR triplet (see Fig. 4 and the discussion in Wang et al. 2008a). These features are common to almost all SNe Ia, although with different strength and duration (Wang et al. 2003; Mazzali et al. 2005a). In the earliest spectrum of SN 2006X (day -10), the photospheric component $\left(\sim 16000 \mathrm{~km} \mathrm{~s}^{-1}\right)$ is accompanied by a much broader HVF at about $28000 \mathrm{~km} \mathrm{~s}^{-1}$, with material expanding at terminal velocities as high as $\sim 40000 \mathrm{~km} \mathrm{~s}^{-1}$, similar to that seen in SN 2002dj (see Mazzali et al. 2005a, their Fig. 2). As in the case of the Si II 6355 Å line, SN 2006X shows much higher Ca II velocities than SN 2002bo (Fig. 4, dotted curve), for which Mazzali et al. (2005a) have identified a HVF at about $20000 \mathrm{~km} \mathrm{~s}^{-1}$.

While discussing the type Ia SN $2005 \mathrm{cg}$, Quimby et al. (2006b) have pointed out a sharp cutoff in the blue wing of the Si II 6355 A line, which they interpreted as the truncation in the velocity distribution generated when the outer ejecta are decelerated by the interaction with CSM. They also noticed that the cutoff velocity appears to align with the blue edge of the Ca II high velocity absorption minimum, supporting the model presented by Gerardy et al. (2004), who attribute these HVFs to a surrounding, H-rich region swept up by the SN ejecta. Quimby et al. (2006b) have also shown that this is a common behavior among spectroscopically normal Ia.

A similar analysis indicates that this is possibly the case also for SN 2006X, which however presents a smoother roll-over from the Si II line blue wing to the continuum than SN $2005 \mathrm{cg}$ (Fig. 5). Even though the blue wing of the Si II line is probably disturbed by an adjacent feature, ten days before maximum light it appears to extend up to $-27000 \mathrm{~km} \mathrm{~s}^{-1}$, which coincides with the blue side of the minimum of the Ca II absorption trough. Nine days later, the two features have a similar profile (see Fig. 5).

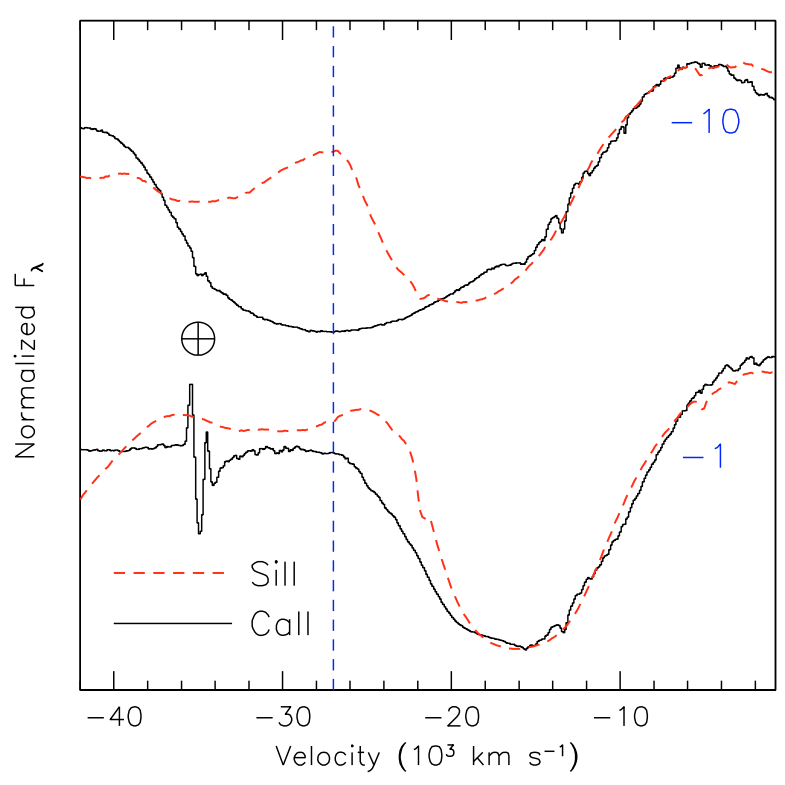

Fig. 5. Si II and Ca II line profiles on day -10 (top) and -1 (bottom). The vertical dashed line marks the Si II cutoff velocity for day -10 .

In conclusion, from a spectroscopic point of view SN 2006X appears as a normal object, even though it has shown expansion velocities which are amongst the highest ever observed for a type Ia $\mathrm{SN}$.

\section{Interstellar polarization and extinction law}

This $\mathrm{SN}$ is known to suffer from substantial extinction (Quimby et al. 2006a; Lauroesch et al. 2006; Wang et al. 2008a) and, therefore, a significant interstellar polarization (hereafter ISP) is expected (Serkowski et al. 1975).

The high resolution spectroscopy (Lauroesch et al. 2006; Patat et al. 2007; Cox \& Patat 2008) has revealed a system of clouds within the host galaxy, and the presence of a distinct molecular cloud with an exceptionally intense $B^{2} \Sigma-X^{2} \Sigma \mathrm{CN}$ vibrational band, accompanied by $\mathrm{CH}, \mathrm{CH}^{+}, \mathrm{Ca} \mathrm{I}$ and diffuse interstellar bands. Stars having comparable features in our own Galaxy are known to have color excesses larger than $E_{B-V}=1$ (Crutcher 1985). Additionally, a small contribution to the extinction is produced by the Milky Way $\left(A_{B} \simeq 0.1\right.$, Schlegel et al. 1998). Given the strong correlation between extinction and linear polarization (Serkowski et al. 1975), a significant amount of ISP is expected for SN 2006X. This is indeed the case, as clearly shown in Fig. 6, where we have combined the data sets of the last two epochs ( +39 and +40 days) to increase the signal-to-noise ratio. With the only remarkable exception being the feature corresponding to the absorption minimum of the near-IR CaII triplet (see next section), there is a rather strong continuum polarization, which steadily decreases from $\sim 8 \%$ at $4000 \AA$ to $\sim 3 \%$ at $8500 \AA$, as already reported by Wang et al. (2006b). This wavelength dependence of the polarization degree is rather different from what is seen in highly reddened stars in our own Galaxy (Serkowski et al. 1975). Although it is possible to fit the data with the empirical Serkowski law:

$$
P(\lambda)=P_{\max } \exp \left(-K \ln ^{2} \frac{\lambda_{\max }}{\lambda}\right)
$$

the resulting values of $K(1.3 \pm 0.1)$ and $\lambda_{\max }(3500 \pm 100 \AA)$ are not compatible with the relation found by Witthet et al. (1992 for 


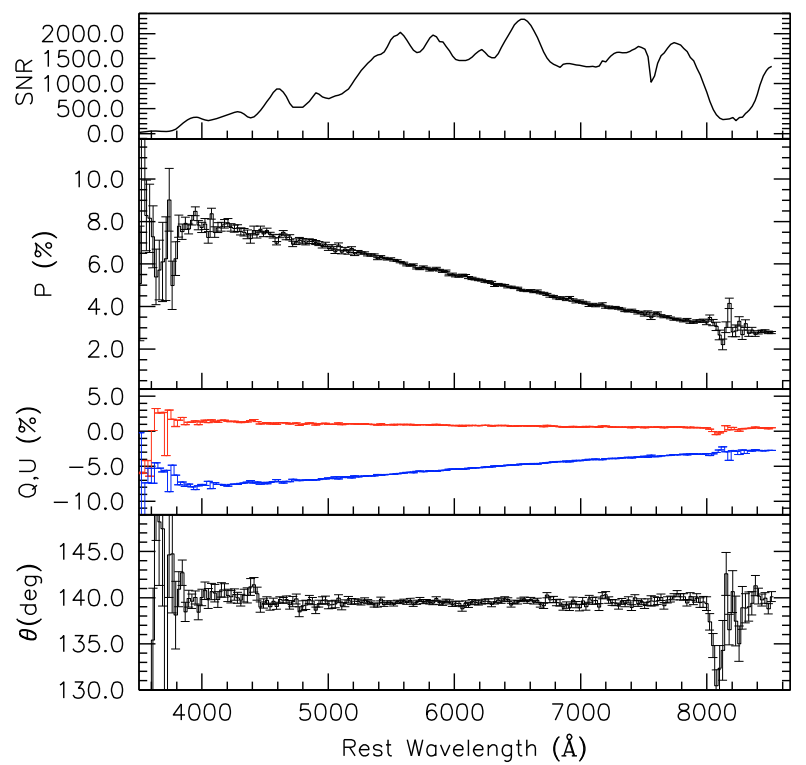

Fig. 6. Spectropolarimetric data of SN 2006X for the last epoch $(+39.2)$. From top to bottom: signal-to-noise ratio (SNR) on the flux spectrum, linear polarization degree $(\mathrm{P})$, Stokes parameters $(\mathrm{Q}, \mathrm{U})$ and polarization angle $(\theta)$. The errorbars indicate 1-sigma level statistical errors.

Galactic stars $\left(K=0.01+1.66 \lambda_{\max }\right.$, where $\lambda_{\max }$ is expressed in microns). In SN 2006X the wavelength dependency is much steeper (Fig. 7). We interpret this as an indication that the dust mixture is significantly different from that typically found in the Milky Way (see also Wang et al. 2006a). This might well be related to the anomalous strength of the $\mathrm{CN}$ absorption seen in high resolution spectroscopy (Lauroesch et al. 2006; Patat et al. 2007), indicating that the bulk of extinction occurred in a cold molecular cloud (Patat et al. 2007; Cox \& Patat 2008).

Therefore, the ratio of total to selective extinction $R_{V}=$ $A_{V} / E(B-V)$ derived for this $\mathrm{SN}$ must be significantly different from what is commonly measured in the Galaxy. Serkowski et al. (1975) have shown that the observed peak of polarization moves bluewards as $R_{V}$ decreases and this is interpreted in terms of dust grain size (see Whittet 1992, and references therein). Therefore, the ISP law observed in SN 2006X, characterized by a very blue peak maximum, hints at a low value of $R_{V}$. Interestingly, the photometric and spectroscopic analysis by Wang et al. (2008a) and Elias-Rosa et al. (2009, in prep.) give $R_{V}=1.48 \pm 0.06$ $(E(B-V)=1.41 \pm 0.04)$ and $R_{V}=1.7 \pm 0.1(E(B-V)=1.2 \pm 0.3)$, respectively. It is worth emphasizing that polarimetry gives an independent proof that the nature of the intervening dust is different from that of a typical Milky Way mixture. Interestingly, clear deviances from the Galactic Serkowski law have been detected in other SNe (Leonard \& Filippenko 2001; Leonard et al. 2002; Maund et al. 2007) and in the bright optical transient in NGC 300 (Patat et al. 2009).

Contrary to most polarimetric observations of SNe Ia, for which it is rather difficult to estimate the ISP contribution (see for instance Leonard et al. 2005 or Wang et al. 2006b), for SN 2006X this is fairly simple, since the continuum polarization is heavily dominated by the interstellar component. Assuming that the high continuum polarization is generated by intervening interstellar material, the ISP Stokes parameters can be estimated as follows:

$Q_{\mathrm{ISP}}(\lambda)=P_{\mathrm{ISP}}(\lambda) \cos \left(2 \theta_{\mathrm{ISP}}\right)$

$U_{\mathrm{ISP}}(\lambda)=P_{\mathrm{ISP}}(\lambda) \sin \left(2 \theta_{\mathrm{ISP}}\right)$.

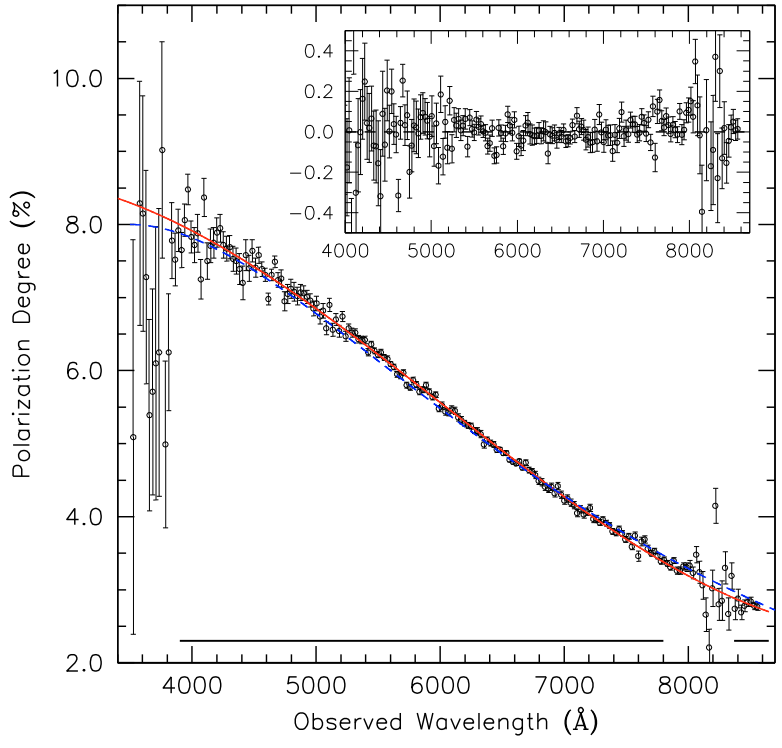

Fig. 7. The degree of linear polarization for the last epoch data (+39 d). The solid curve is a 3 rd order polynomial fit to the observed data in the wavelength ranges indicated by the horizontal segments close to the $x$-axis scale. The dashed curve is a best fit using a Serkowski law $\left(P_{\max }=8.0 \%, \lambda_{\max }=3500 \AA\right.$ and $\left.K=1.30\right)$. The upper right insert shows the residuals from the best fit polynomial relation.

Rather than adopting a Serkowski law for the degree of polarization, we have preferred to use a 3 rd order polynomial fit to the data, performed in the wavelength ranges 3900-7800 $\AA$ and 8350-8650 $\AA$ in order to avoid low signal-to-noise data and to exclude the Ca II IR triplet region, where a polarization signal from the SN is still present. Figure 7 shows the best fit corresponding to the following values for the polynomial coefficients:

$P_{\mathrm{ISP}}(\lambda)=5.6-13.2\left(\lambda-\lambda_{0}\right)-0.1\left(\lambda-\lambda_{0}\right)^{2}+36.9\left(\lambda-\lambda_{0}\right)^{3}$

where $P_{\text {ISP }}$ is in per cent, $\lambda$ is expressed in microns and $\lambda_{0}=0.6 \mu \mathrm{m}$. The rms deviation from the best fit relation is about $0.1 \%$, in line with the measurement accuracy (the statistical rms uncertainties on the polynomial coefficients are 0.02 , $0.16,0.68$, and 4.76 respectively).

Since the polarization position angle $\theta_{\text {ISP }}$ can be considered wavelength independent (see for example Whittet et al. 1992; see also Fig. 6, lower panel), we have estimated it by simply computing the average value within SN line-free regions in our last-epoch spectropolarimetric data (see Fig. 8). This turns out to be $\theta_{\text {ISP }}=139.5 \pm 0.1$ degrees. Using this value, Eqs. (2) and (1), the intrinsic SN polarization can be derived by subtracting $Q_{\mathrm{ISP}}$, $U_{\text {ISP }}$ from the observed $Q, U$ Stokes parameters and recomputing the polarization degree and polarization angle. This correction was applied to all epochs, assuming that the ISP does not change with time. The constancy of the ISP is confirmed by inspection of the observed spectropolarimetric data, which do not show any significant variation in the line-free regions. This is illustrated more quantitatively in Table 2, where we have reported the results of a linear squares fitting performed in the wavelength range $6400 \AA \leq \lambda \leq 7200 \AA$, which is known to be free of strongly polarized SN lines (Kasen et al. 2004; Leonard et al. 2005; Wang et al. 2006b). Besides listing the slope $b$ and its rms uncertainty, Table 2 also presents the rms scatter from the fitted linear law $(\sigma)$ and the polarization degree interpolated at the center of the spectral range $\left(P_{6800}\right)$ and its rms uncertainty. The small fluctuations seen in $P_{6800}$, which reach a peak-to-peak 
Table 2. ISP linear least squares fitting parameters for the wavelength range 6400-7200 ̊.

\begin{tabular}{cccc}
\hline \hline $\begin{array}{c}\text { Phase } \\
\text { (days) }\end{array}$ & $\begin{array}{c}b \\
\left(\% \AA^{-1}\right)\end{array}$ & $\begin{array}{c}\sigma \\
(\%)\end{array}$ & $\begin{array}{c}P_{6800} \\
(\%)\end{array}$ \\
\hline-10 & $-0.0013 \pm 0.00008$ & 0.10 & $4.57 \pm 0.05$ \\
-8 & $-0.0014 \pm 0.00007$ & 0.09 & $4.42 \pm 0.04$ \\
-7 & $-0.0014 \pm 0.00004$ & 0.05 & $4.51 \pm 0.03$ \\
-6 & $-0.0013 \pm 0.00005$ & 0.06 & $4.45 \pm 0.03$ \\
-3 & $-0.0013 \pm 0.00004$ & 0.04 & $4.52 \pm 0.02$ \\
-2 & $-0.0013 \pm 0.00003$ & 0.04 & $4.50 \pm 0.02$ \\
-1 & $-0.0012 \pm 0.00005$ & 0.06 & $4.54 \pm 0.03$ \\
+39 & $-0.0012 \pm 0.00005$ & 0.04 & $4.51 \pm 0.02$ \\
\hline
\end{tabular}

value of $0.15 \%$, are fully consistent with a null variation within the measured errors.

Since most of the extinction takes place in what is probably a cold molecular cloud (Patat et al. 2007; Cox \& Patat 2008), we argue this is true for the ISP $^{2}$. During the epochs covered by our observations, that span about 50 days, several narrow $\mathrm{Na}$ I D features have shown a significant evolution, which has been interpreted as due to changes in the physical conditions of circumstellar material induced by the $\mathrm{SN}$ radiation field and/or direct interaction with the fast expanding SN ejecta (Patat et al. 2007). In contrast, no variations have been detected in the $\mathrm{Na} \mathrm{I}$, $\mathrm{Ca}$ II, $\mathrm{CN}, \mathrm{CH}, \mathrm{CH}^{+}$and $\mathrm{Ca}$ I components associated with the molecular cloud.

In principle, if the CSM material is rich in dust and provided that dust can survive the $\mathrm{SN}$ radiation field, changes in its physical conditions might be reflected in variations in the extinction suffered by the SN. Of course, such changes are difficult to disentangle from other effects on the photometry, because of the intrinsic SN color evolution, the heavy reddening and the presence of a light echo (Wang et al. 2008a, 2008b; Crotts \& Yourdon 2008). On the other hand, the ISP is so strong in SN 2006X in comparison to what is expected for the intrinsic SN continuum polarization that the effects of the $\mathrm{SN}$ evolution are negligible. This makes spectropolarimetry a promising tool to reveal variations in the conditions of the obscuring material. As we have just shown, the ISP degree appears to be constant within the measurement errors. This suggests that the bulk of polarization takes place within the molecular cloud, which is undisturbed by the $\mathrm{SN}$ explosion.

The derived ISP position angle appears to be tangential to the dust lane associated to the spiral arm close to the explosion site (see Fig. 1). This is perfectly in line with the well known fact that dust grains tend to be aligned along the magnetic field, which follows rather closely the spiral pattern of galaxies (see for example Scarrot et al. 1987). The same behavior was seen, for instance, in the ISP deduced from the spectropolarimetric data of SNIa 2001el (Wang et al. 2003).

The fact that a light echo has been detected in SN 2006X (Wang et al. 2008b; Crotts \& Yourdon 2008) suggests that an additional, possibly time-dependent source of continuum polarization might be present. In fact, if the distribution of the scattering dust is asymmetric with respect to the line of sight, this is expected to produce a net, non-null continuum polarization (Wang \& Wheeler 1996b; Patat 2005) and broad polarized spectral features (Wang \& Wheeler 1996b). However, while the polarized flux is supposed to be high when the scattered light dominates

\footnotetext{
2 The column densities associated with the time-variant IS features are two orders of magnitude lower than that deduced for the saturated components.
}

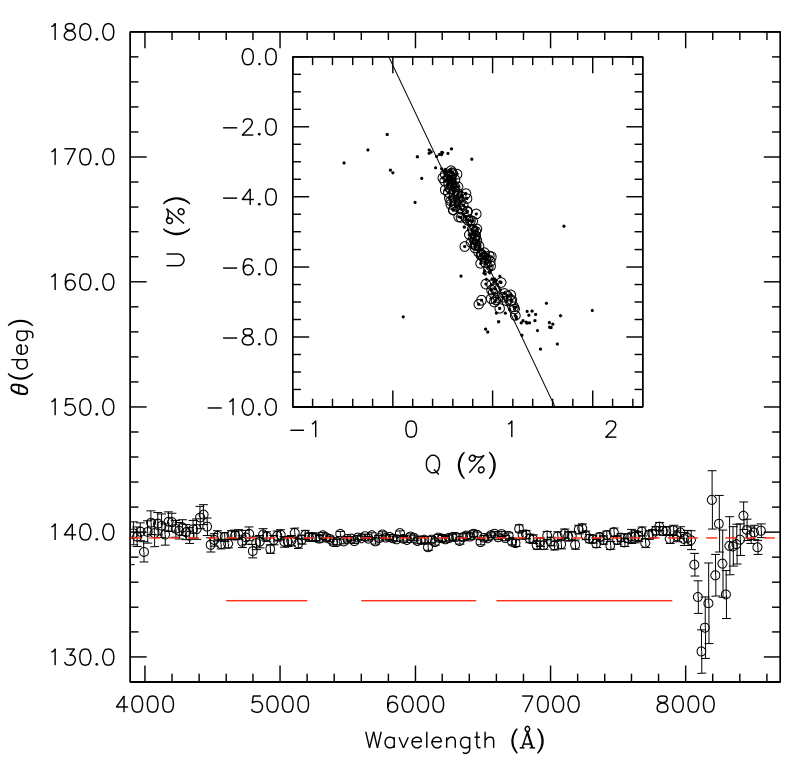

Fig. 8. Linear polarization position angle for the last epoch. The dashed line is placed at the average value computed within the wavelength ranges indicated by the three horizontal segments. The insert shows the observed $Q-U$ plane. Circles mark data within the selected wavelength ranges, while the straight line is a best fit to this data subset.

over the light directly emitted by the SN (i.e. at late epochs), that should not be the case around maximum light, when the polarized photons are extremely diluted into the basically unpolarized radiation emitted by the photosphere. This additional effect can be quantified only by detailed modeling. Here we notice that since the ISP aligns with the spiral pattern of NGC 4321, it is most probable that the bulk of polarization arises in a cloud belonging to the disk of the host and hence has nothing to do with the CS environment of SN 2006X.

\section{Intrinsic polarization}

The spectropolarimetric evolution of SN 2006X on the eight epochs covered by our observations is summarized in Fig. 9, (more detailed data for each single epoch are presented in Figs. A.1 to A.8).

As is generally the case for $\mathrm{SNe}$ (Cropper et al. 1988), the polarization signal is present in rough coincidence with the minima of P-Cygni profiles. Moreover, as for other SNe Ia (Wang et al. 2003), the most prominent features are observed in Si II $6355 \AA$ and the near-IR Ca II triplet. This is clearly illustrated for day -2 in Fig. 10, where we have also given tentative identifications for the main features, following the case of SN 2004dt (Wang et al. 2006b).

The evolution of most prominent features will be discussed in the next subsections and here we just give a general overview of the spectropolarimetric evolution.

Starting with the first spectrum, ten days before maximum light, the most prominent feature is the Ca II IR triplet, which shows a maximum polarization as high as $\sim 1.5 \%$. A lower polarization signal is detected for the Si II $6344 \AA$ line, which displays a maximum polarization of $\sim 0.5 \%$. Even though disturbed by a rather high noise, other features in the blue spectral range, like the Si II $5051 \AA$ and the Mg II $4471 \AA$, exhibit low polarization levels $(\leq 0.5 \%)$. Also, the continuum between 6200 and $7000 \AA$ shows a low polarization level, of about $0.2 \%$, compatible with the values reported for SNe 2001el (Wang et al. 2003) 


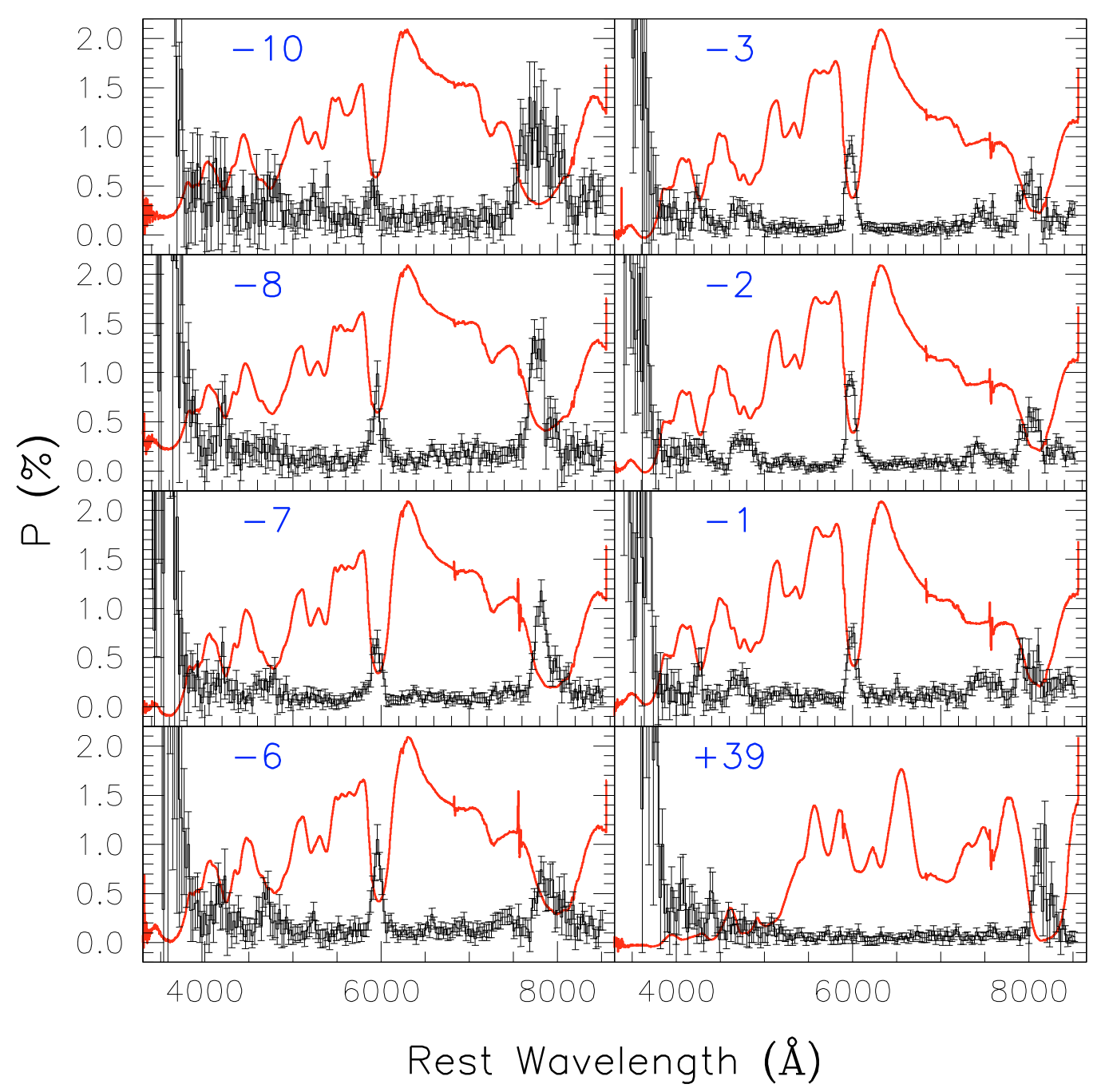

Fig. 9. Spectropolarimetric evolution of SN 2006X. For each epoch the ISP-corrected polarization degree is presented together with the unbinned flux spectrum (solid line). Epochs refer to $B$ maximum light.

and 2004dt (Wang et al. 2006b; Leonard et al. 2005). As time goes by, the Ca II IR triplet polarization decreases and this is accompanied by a quite rapid increase in the polarization level of the Si II $6355 \AA$ feature, which reaches a peak value of $\sim 1.1 \%$ on day -6 and decreases again to reach $\sim 0.8 \%$ on day -1 . This roughly conforms to the behavior reported by Wang et al. (2007) for SN 2002bo, which reached the Si II polarization peak about at the same epoch.

On day -1 the $\mathrm{Ca}$ II polarization appears to be reduced to $0.63 \pm 0.17$. Finally, on the last epoch (day +39$)$, no trace of Si II polarization is seen and the only detectable lines are the Ca II IR triplet $(\sim 1 \%)$ and some unidentified features between 4000 and $6000 \AA$. The continuum in the 6000-7000 region shows a very low polarization level $(0.1 \%)$ which, given the measurement errors, is consistent with a null polarization and lends some support to the applied ISP correction (see Sect. 4). The apparent re-polarization of the Ca II IR triplet detected on day +39 will be discussed in Sect. 5.2.

In general, SN 2006X has an intermediate behavior between SN 2001el (Wang et al. 2003), for which the Ca II IR triplet was more polarized than the Si II $6355 \AA$ line, and SN 2004dt (Wang et al. 2006b), which showed the opposite. Also, the latter object has shown a lower continuum polarization level, while the former was more polarized than SN 2006X. Despite the strong signal detected in several spectral features, SN 2004dt did not show any trace of polarization corresponding to the O I 7774 line (Wang et al. 2006b; Leonard et al. 2005). This is also the case for SN 2006X (see Figs. 9, 10 and Sect. 5.3), for which the O I polarization is certainly lower than $0.3 \%$ at any epoch.

Before proceeding with the detailed study of the most prominent features, it is interesting to analyze the polarization on the plane of the Stokes $Q-U$ parameters. The presence of a dominant direction can often be identified through the geometric distribution of points in this plane (see for example Wang et al. 2001; Wang \& Wheeler 2008). For this purpose, following Wang et al. (2003), we have performed a weighted linear squares fit to the data using the linear relation $U=\alpha+\beta Q$. The position angle of the dominant axis is then given by $\theta_{d}=1 / 2 \arctan \beta$. We have done this for each epoch in the wavelength range $4000 \leq \lambda \leq 8600 \AA$. The results are plotted in Fig. 11 and summarized in Table 3, where we have also reported the uncertainties on $\alpha, \beta$ and $\theta_{d}$, the rms deviation $\sigma$ from the best fit relation and the probability $p$ of chance linear correlation (low values of $p$ indicate a significant correlation). Of course, the relation between $\theta_{d}$ and $\beta$ rigorously holds only if the best fit axis goes through the origin of the $Q-U$ coordinates system. As one 


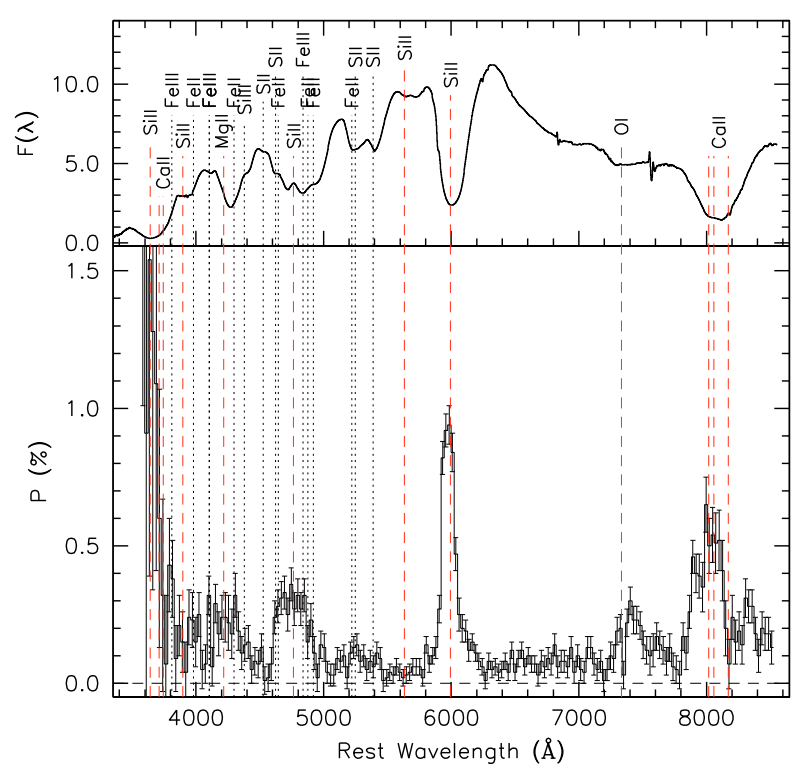

Fig. 10. Spectropolarimetry of SN 2006X on day -2. Main line identifications are given in the upper panel. Vertical dashed lines correspond to an expansion velocity of $17000 \mathrm{~km} \mathrm{~s}^{-1}$, while the dotted lines correspond to $11000 \mathrm{~km} \mathrm{~s}^{-1}$.

Table 3. Dominant axis weighted fittings for the eight epochs.

\begin{tabular}{ccccccc}
\hline \hline $\begin{array}{c}\text { Phase } \\
\text { (days) }\end{array}$ & $\begin{array}{c}\alpha \\
(\%)\end{array}$ & $\beta$ & $\chi^{2}$ & $\begin{array}{c}\sigma \\
(\%)\end{array}$ & $\begin{array}{c}\theta_{d} \\
(\mathrm{deg})\end{array}$ & $p$ \\
\hline-10 & $-0.082(0.011)$ & $-0.158(0.058)$ & 300 & 0.28 & $85.5(3.3)$ & 0.18 \\
-8 & $+0.084(0.007)$ & $-0.269(0.040)$ & 291 & 0.15 & $82.5(2.3)$ & $<10^{-8}$ \\
-7 & $+0.040(0.004)$ & $-0.354(0.032)$ & 376 & 0.12 & $80.3(1.8)$ & $<10^{-8}$ \\
-6 & $+0.082(0.006)$ & $-0.274(0.045)$ & 262 & 0.14 & $85.4(2.5)$ & $<10^{-8}$ \\
-3 & $-0.002(0.004)$ & $-0.404(0.035)$ & 514 & 0.14 & $79.0(2.0)$ & $<10^{-8}$ \\
-2 & $+0.042(0.003)$ & $-0.362(0.027)$ & 706 & 0.13 & $80.1(1.5)$ & $<10^{-8}$ \\
-1 & $-0.015(0.006)$ & $-0.363(0.042)$ & 401 & 0.14 & $80.0(2.3)$ & $<10^{-8}$ \\
+39 & $-0.003(0.004)$ & $-0.018(0.063)$ & 275 & 0.16 & $89.5(3.6)$ & 0.73 \\
\hline
\end{tabular}

Note: data with $4000 \leq \lambda \leq 8600 \AA$ were used. The number of degrees of freedom is 175 .

can see from the values of $\alpha$ in Table 3, this is true to a good approximation (see also Fig. 11).

The first thing to notice is that on the first and on the last epoch there is no clear dominant axis (the probability of chance correlation is high, see Table 3 ). On day -10 this is probably due to the relatively large errorbars on the data, while on day +39 this is most likely related to the intrinsic low polarization. With the exception of these two cases, a dominant axis seems to be present, almost parallel to the $Q$ axis: the weighted average position angle is $\left\langle\theta_{d}\right\rangle=+82.0 \pm 0.8$ degrees. Even though the data points appear to get closer and closer to the dominant axis as the SN approaches maximum light, significant departures are visible. SN 2006X would be classified as spectropolarimetry type D1 in the terminology of Wang \& Wheeler (2008) corresponding to a distinct dominant axis, but also significant departures from that axis. Individual lines would be classed as SP type L for loops, as discussed in the next sections.

\subsection{The Si II 6355 Å line}

At the first epoch, the polarization of Si II $6355 \AA$ line peaks at $\sim 0.57 \pm 0.19 \%$ (see Fig. 12). In the subsequent days the
Table 4. Si II dominant axis weighted fittings for the eight epochs.

\begin{tabular}{ccccccc}
\hline \hline $\begin{array}{c}\text { Phase } \\
\text { (days) }\end{array}$ & $\begin{array}{c}\alpha \\
(\%)\end{array}$ & $\beta$ & $\chi^{2}$ & $\begin{array}{c}\sigma \\
(\%)\end{array}$ & $\begin{array}{c}\theta_{d} \\
(\mathrm{deg})\end{array}$ & $p$ \\
\hline-10 & $+0.024(0.042)$ & $-0.192(0.213)$ & 19 & 0.20 & $84.6(12.1)$ & 0.55 \\
-8 & $+0.131(0.034)$ & $-0.625(0.162)$ & 22 & 0.15 & $74.0(8.6)$ & $8 \times 10^{-3}$ \\
-7 & $+0.099(0.019)$ & $-0.509(0.095)$ & 21 & 0.11 & $76.5(5.2)$ & $9 \times 10^{-3}$ \\
-6 & $+0.110(0.032)$ & $-0.687(0.124)$ & 13 & 0.12 & $72.8(6.5)$ & $2 \times 10^{-4}$ \\
-3 & $+0.043(0.021)$ & $-0.576(0.074)$ & 81 & 0.20 & $75.0(4.0)$ & 0.02 \\
-2 & $+0.115(0.015)$ & $-0.401(0.050)$ & 141 & 0.21 & $79.1(2.8)$ & 0.04 \\
-1 & $+0.064(0.021)$ & $-0.443(0.095)$ & 54 & 0.18 & $78.1(5.2)$ & 0.03 \\
+39 & $-0.010(0.012)$ & $-0.318(0.235)$ & 14 & 0.05 & $81.2(13.1)$ & 0.47 \\
\hline
\end{tabular}

Note: data with velocities between -25000 and $-10000 \mathrm{~km} \mathrm{~s}^{-1}$ were included. The number of degrees of freedom is 11 .

polarization degree increases and the polarization peak gets narrower and, at variance with the Ca II IR triplet (see next section), it is only very slightly blue-shifted with respect to the line absorption minimum. Maximum peak polarization is reached on day $-6(1.05 \pm 0.14 \%)$, and in the following days it slightly decreases, to reach $0.75 \pm 0.10 \%$ on day -1 . Finally, on our last epoch (+39) the polarization is $\leq 0.15 \%$.

A dominant axis is clearly present for the Si II line on day -7 (see Fig. 13, upper panel), at an average position angle $\theta=70.4 \pm 8.2$ degrees. This value, which was computed in the velocity range $-23000 \leq v \leq-17000 \mathrm{~km} \mathrm{~s}^{-1}$, is consistent at the $1-\sigma$ level with the one deduced for Ca II $(78.0 \pm 3.4$; see next section) and at the 2- $\sigma$ level with the overall angle as given in Table 3 and illustrated in Fig. 11. Following Wang et al. (2003), we computed the components of the Si II polarization along $\left(P_{d}\right)$ and perpendicular $\left(P_{0}\right)$ to the dominant axis, which are obtained by rotating counterclockwise the observed $Q-U$ coordinates system by the angle $\theta$ that defines the dominant axis.

The Si II polarization is practically parallel to the dominant axis, i.e. $P_{0} \approx 0$ (see Fig. 13, lower panel), at variance with what is seen for Ca II (see next section). A hint of a dominant axis is still present on day -1 , at least for the core of the absorption profile and with a lower value for the position angle (see Fig. 14). Nevertheless, $Q-U$ diagrams show significant deviations and the presence of clear loops cut through by the dominant axis (Fig. 15).

The results of a weighted linear squares fit in the Si II $Q-$ $U$ plane are presented in Table 4 (see also Sect. 5).

\subsection{The Ca II IR triplet}

As in the case of the well studied SN 2001el (Wang et al. 2003), the Ca II IR triplet is the most prominent feature seen during the very early phases. Its polarimetric evolution is presented in Fig. 16. As already mentioned in Sect. 3, this feature shows extremely high expansion velocities, giving evidence of material moving as fast as $40000 \mathrm{~km} \mathrm{~s}^{-1}$ on day -10 (see Fig. 4). At this epoch, the minimum of the absorption trough is at about $27000 \mathrm{~km} \mathrm{~s}^{-1}$, which is much higher than the expansion velocity deduced for the Si II $6355 \AA$ absorption minimum at the same epoch, i.e. $19500 \mathrm{~km} \mathrm{~s}^{-1}$.

Most likely the Ca II line profile is a signature of a HVF (Mazzali et al. 2005a; Gerardy et al. 2004, and references therein), of the type discovered in SN 2001el (Wang et al. 2003), even though in that case the HVF was clearly detached from the photospheric component (Wang et al. 2003; Kasen et al. 2003). Due to the much higher expansion velocities measured in SN 2006X, the line broadening causes a significant 

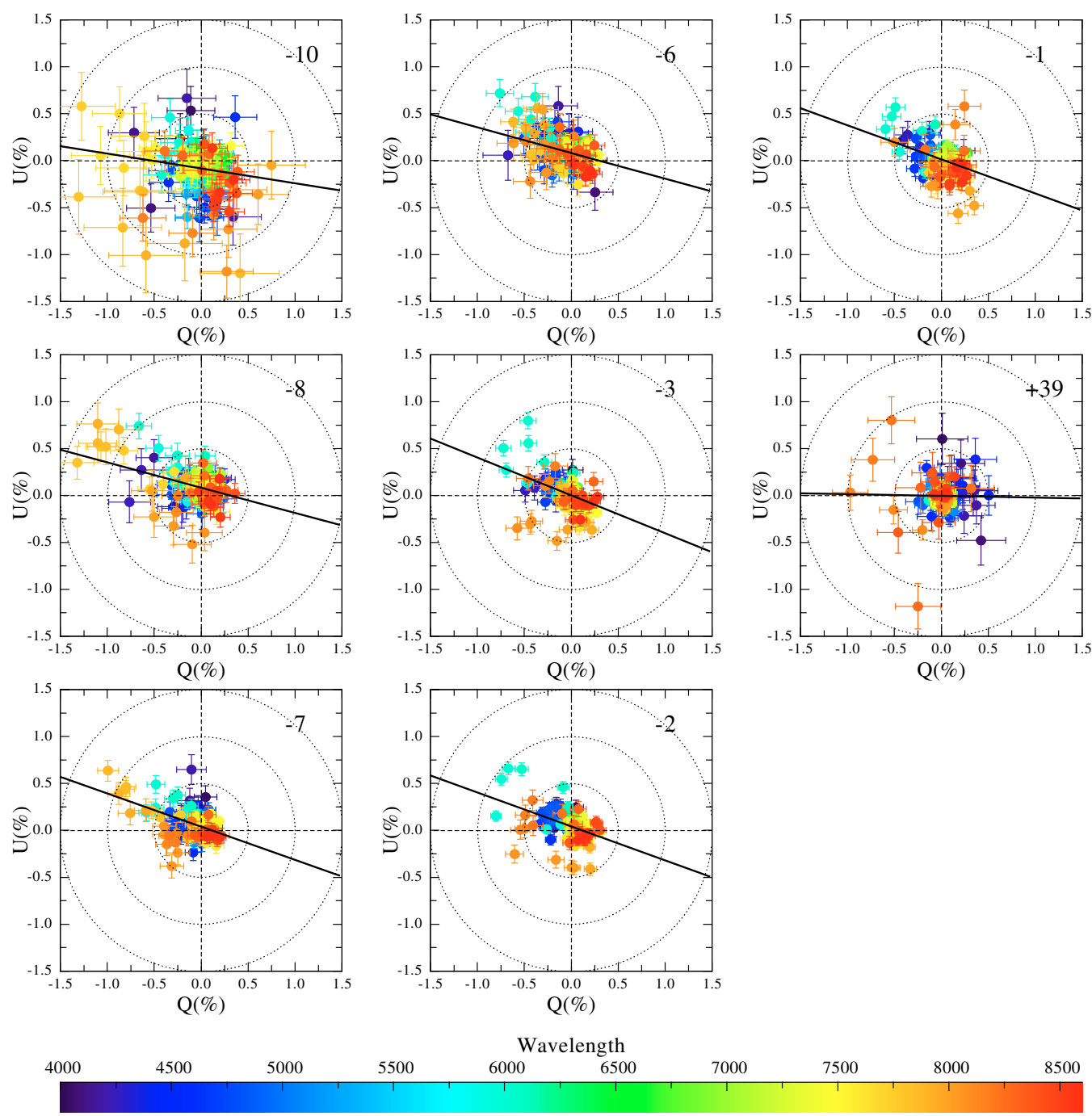

Fig. 11. ISP-corrected spectropolarimetric data of SN 2006X on the $Q-U$ plane as a function of wavelength for all epochs (indicated on the upper right corner of each panel). The straight line traces the dominant axis derived from a weighted least squares fit (see Sect. 5), while the three dotted circles indicate $0.5,1.0$ and $1.5 \%$ polarization levels.

blending. In general, the polarization profile is non-homomorph with respect to the flux profile, with the polarization peak being systematically bluer than the absorption center. This is better seen in Fig. 17, where we present the data for day -7 . The polarization peaks at about $-26000 \mathrm{~km} \mathrm{~s}^{-1}(1.18 \pm 0.11 \%)$ while the expansion velocity deduced from Si II $6355 \AA$ on the same epoch is $\sim 16600 \mathrm{~km} \mathrm{~s}^{-1}$. The photospheric component of $\mathrm{Ca}$ II shows a polarization signal, that is definitely lower than in the HVF $(0.34 \pm 0.11 \%)$. As shown by the upper panel of Fig. 17, the position angle within the HVF shows relatively small variations: the average value computed in the velocity range $-30000 \leq v \leq-24000 \mathrm{~km} \mathrm{~s}^{-1}$ is $\langle\theta\rangle=+78.0 \pm 3.4$ degrees, which is actually not very far from the dominant axis position deduced from the whole data sets $(82.0 \pm 0.8)$. The photospheric component shows no clear dominant axis (see Fig. 17).

The result of the $P_{d}-P_{0}$ decomposition is shown in the lower panel of Fig. 17. As expected, $P_{0} \approx 0$ within the HVF, while $P_{d}$ and $P_{0}$ contribute in a similar way to the polarization of the photospheric component, suggesting that the matter concerned is clumpy (Wang et al. 2003; Tanaka et al. 2006).

Another interesting fact is the rapid time evolution. As the SN approaches maximum light things change rather dramatically. On day -2 (see Fig. 18) the minimum of the absorption trough corresponds to a velocity which is very close to the one indicated by the Si II $6355 \AA$ feature $\left(-16600 \mathrm{~km} \mathrm{~s}^{-1}\right)$. The polarization still peaks at higher velocities, even though the peak is not very well defined and two different features seem to co-exist. One, centered at about $-18000 \mathrm{~km} \mathrm{~s}^{-1}(0.7 \pm 0.1 \%)$, shows a dominant axis, not too far from the one identified in the previous epoch and actually very close to the average dominant axis defined by the whole data set. The higher velocity component, peaking around $-24000 \mathrm{~km} \mathrm{~s}^{-1}$, has a slightly lower polarization $(\sim 0.5 \pm 0.1 \%)$ but a definitely different orientation: even though the position angle changes gradually across the feature (Fig. 18, upper panel), on average it is practically orthogonal to the slower component. This is shown also by the $P_{d}-P_{0}$ decomposition (Fig. 18, lower panel).

In general, the Ca II polarization shows significant deviations from a dominant axis. This is illustrated in Fig. 20, where we present $Q-U$ diagrams for all epochs, including data in the velocity range $-40000 \leq v \leq-10000 \mathrm{~km} \mathrm{~s}^{-1}$. With only the exception of the first epoch, the best-fit axis is never too far from the average dominant axis, even though pronounced loops and a quite erratic behavior are clearly seen. The results of a weighted 


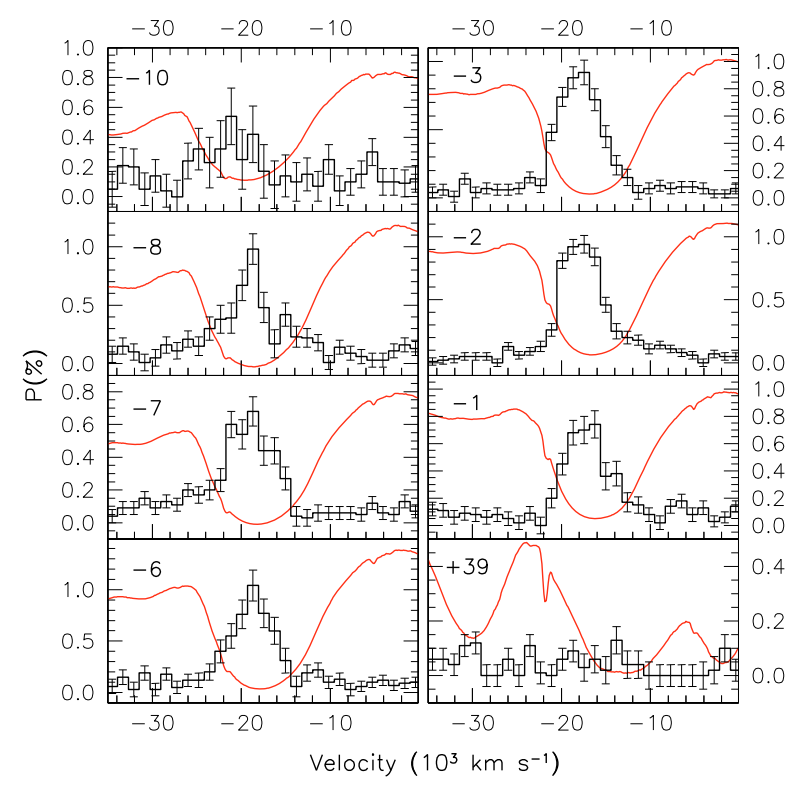

Fig. 12. Polarimetric evolution of the Si II $6355 \AA$ line profile. The smooth solid curve traces the unbinned flux spectrum, arbitrarily scaled for presentation. Note the different vertical scales in each plot. The weak feature around $-22000 \mathrm{~km} \mathrm{~s}^{-1}$ is due to IS Na I D absorption.

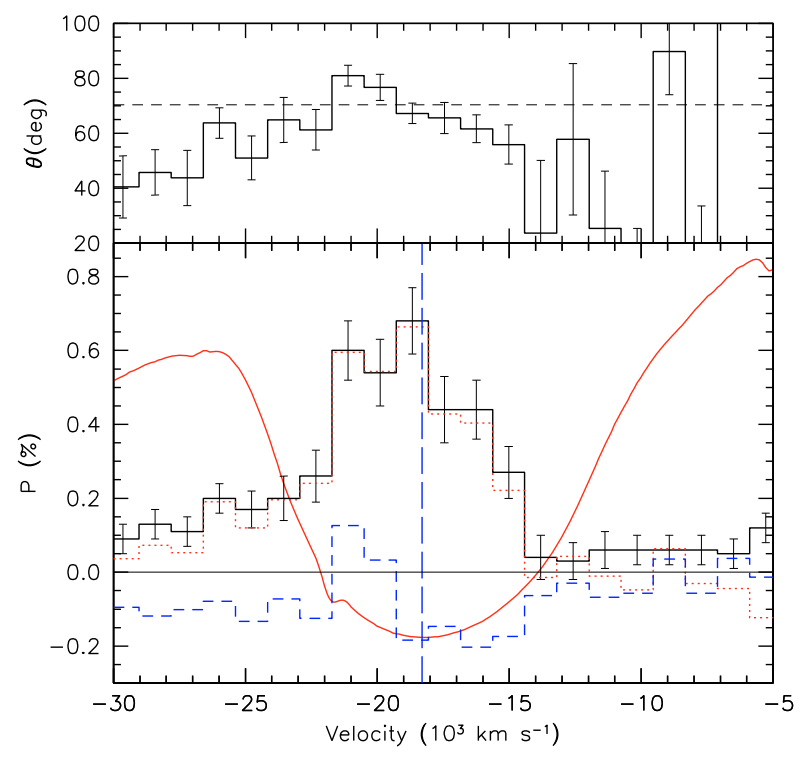

Fig. 13. Lower panel: polarization profile of Si II $6355 \AA$ on day -7 . Dotted and dashed lines trace $P_{d}$ and $P_{0}$ respectively (see Sect. 5.1). The vertical dashed line indicates the expansion velocity deduced from Si II $6355 \AA$ absorption minimum for this epoch. Upper panel: polarization position angle. The horizontal dashed line indicates the average angle computed in the velocity range $-23000 \leq \mathrm{v} \leq-17000 \mathrm{~km} \mathrm{~s}^{-1}$. The weak absorption feature around $-22000 \mathrm{~km} \mathrm{~s}^{-1}$ is due to IS Na I D.

linear squares fit in the $Q-U$ plane are presented in Table 5 (see also the introductory part of Sect. 5).

Finally, there is an interesting fact that concerns the last epoch of our data set. At variance with the well studied SN 2001el (Wang et al. 2003), which had shown no detectable trace of polarization in the Ca II IR triplet on day +41 (bearing in mind that the signal-to-noise ratio was lower in that case), and SN 2004du that displayed a very low polarization level $(\sim 0.2 \%) 18$ days after maximum light (Leonard et al. 2005),
Table 5. Ca II dominant axis weighted fittings for the eight epochs.

\begin{tabular}{ccccccc}
\hline \hline $\begin{array}{l}\text { Phase } \\
\text { (days) }\end{array}$ & $\begin{array}{c}\alpha \\
(\%)\end{array}$ & $\beta$ & $\chi^{2}$ & $\begin{array}{c}\sigma \\
(\%)\end{array}$ & $\begin{array}{c}\theta_{d} \\
(\mathrm{deg})\end{array}$ & $p$ \\
\hline-10 & $-0.224(0.045)$ & $-0.264(0.095)$ & 59 & 0.43 & $82.6(5.4)$ & 0.06 \\
-8 & $+0.041(0.025)$ & $-0.335(0.058)$ & 55 & 0.24 & $80.7(3.3)$ & $3 \times 10^{-5}$ \\
-7 & $-0.012(0.015)$ & $-0.345(0.048)$ & 89 & 0.17 & $80.5(2.7)$ & $3 \times 10^{-5}$ \\
-6 & $+0.038(0.021)$ & $-0.293(0.080)$ & 59 & 0.19 & $81.9(4.5)$ & 0.02 \\
-3 & $-0.103(0.013)$ & $-0.157(0.088)$ & 129 & 0.19 & $85.6(5.0)$ & 0.82 \\
-2 & $-0.078(0.011)$ & $-0.190(0.067)$ & 143 & 0.17 & $84.6(3.8)$ & 0.11 \\
-1 & $-0.105(0.021)$ & $-0.298(0.119)$ & 82 & 0.22 & $81.7(6.7)$ & 0.33 \\
+39 & $-0.030(0.010)$ & $+0.032(0.106)$ & 88 & 0.30 & $90.9(6.1)$ & 0.62 \\
\hline
\end{tabular}

Note: data with velocities between -40000 and $-10000 \mathrm{~km} \mathrm{~s}^{-1}$ were included. The number of degrees of freedom is 31 .

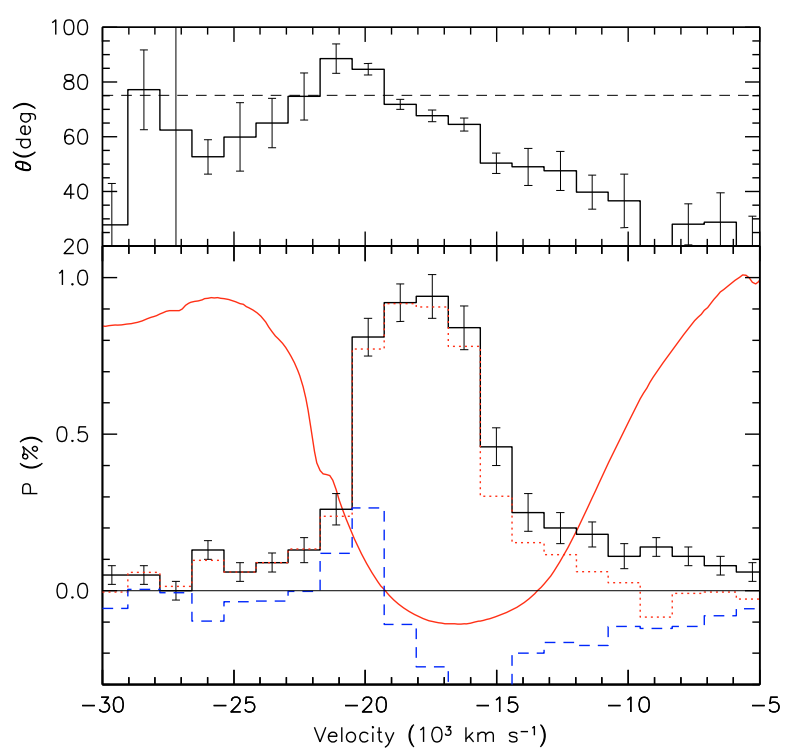

Fig. 14. Same as Fig. 13 for day -2 . The horizontal dashed line in the upper panel indicates the dominant axis deduced on day -7 .

SN 2006X presents a significant signal at a comparably late epoch $($ day +39$)$. The polarized feature, centered at about $-16000 \mathrm{~km} \mathrm{~s}^{-1}$, reaches $1.20 \pm 0.24 \%$ (see Fig. 19). Even though the SN was already at $V \sim 16$ at these phases (see Table 1), as a result of the $2 \mathrm{~h}$ total integration time the signal-to-noise ratio in the combined binned flux spectrum is about 340 at the minimum of the Ca II absorption trough (see Fig. 6, upper plot), so that the detected polarized feature, reaching a 5$\sigma$ level, is real. The data do not show evidence for a dominant axis; on the contrary, the polarization angle shows a smooth variation across the absorption (Fig. 19), which turns into a loop on the $Q-U$ plane (Fig. 20). An important aspect to note is that the high-velocity component visible on day -1 at about $-24000 \mathrm{~km} \mathrm{~s}^{-1}$ (Fig. 16) has completely disappeared on day +39 , while the other $\left(-16000 \mathrm{~km} \mathrm{~s}^{-1}\right)$ has grown in polarization and possibly receded in velocity. As one can see in Fig. 19, the emergence of this polarized line is accompanied by a substantial broadening of the absorption trough in the flux spectrum on the red side. A lower polarization $(<0.5 \%)$ is seen also around $-10000 \mathrm{~km} \mathrm{~s}^{-1}$ but, given the uncertainties, this is only marginally significant and is consistent with a null polarization at the $\sim 2.5-\sigma$ level. 

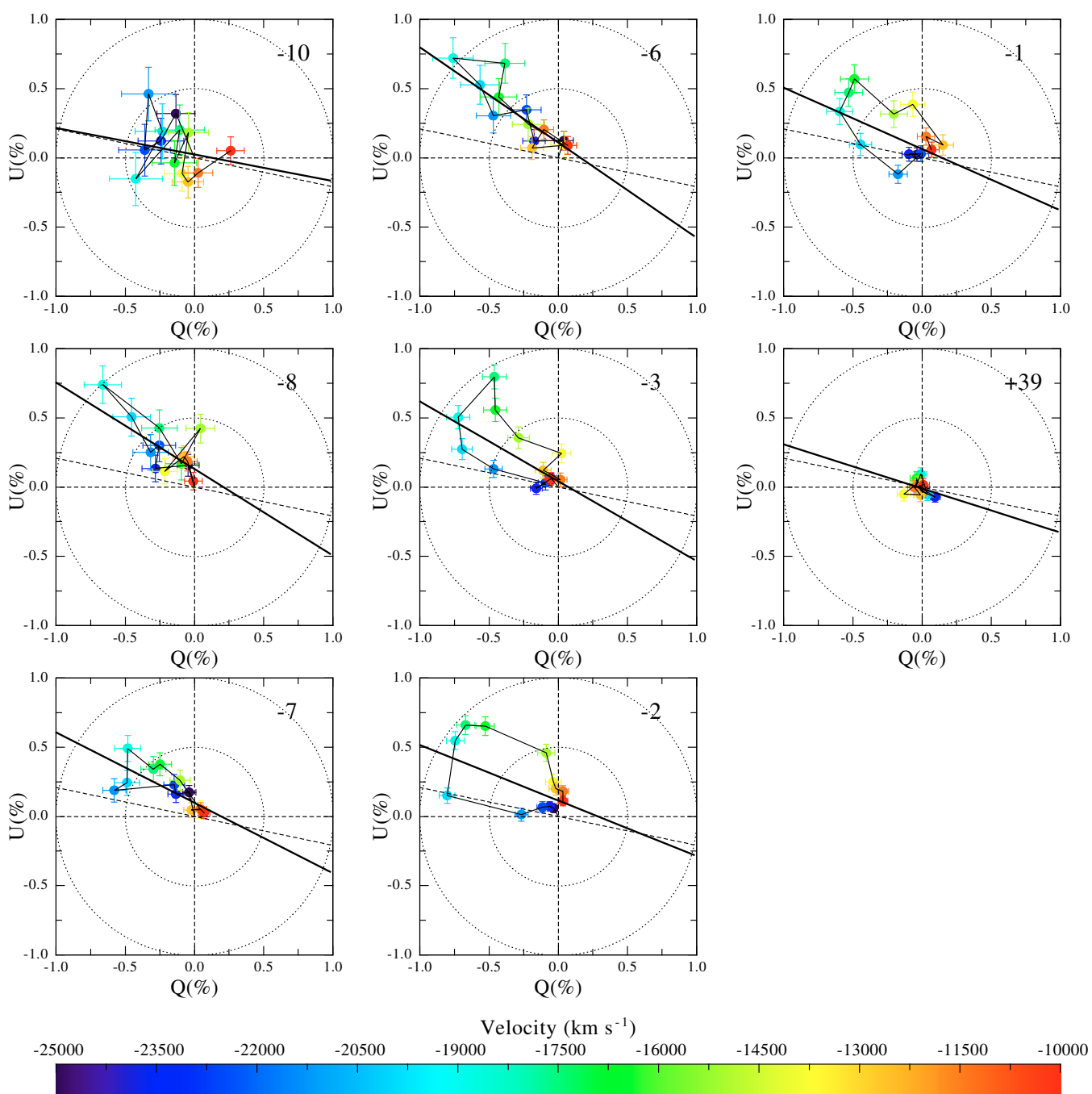

Fig. 15. $Q-U$ diagrams for Si II $6355 \AA$. The dotted line traces the average dominant axis at $\left\langle\theta_{d}\right\rangle=+82.0$ degrees (see Sect. 5), while the solid line is the linear weighted fit to the displayed Si II data.

\subsection{The O I $7774 \AA$ line and other minor features}

The O I $7774 \AA$ does not show any detectable polarization (see Fig. 21), as in SN 2004dt (Wang et al. 2006b). At all epochs the polarization across this line is $\leq 0.2 \%$. Wang et al. (2005, 2006b) have proposed that primordial oxygen is more "spherically" distributed than newly-synthesized silicon, magnesium and calcium. An important part of the argument for the case of SN 2004dt was that the O I and Si II had closely the same velocity profile, i.e. they shared the same velocity space and yet had substantially different polarization. This conclusion holds also for SN 2006X, at least in the earliest epochs, when the O I line can be clearly traced (Fig. 22).

In the framework of hydrodynamical and nucleosynthesis models, the difference in polarization between $\mathrm{O}$ and $\mathrm{Si} / \mathrm{Ca}$ may be understood as a consequence of their sources (see Fig. 23). Oxygen originates from both pre-explosion unburned WD matter in the outer layers and from freshly synthesized $\mathrm{O}$ during explosive $\mathrm{C}$-burning. The corresponding $\mathrm{O}$ abundances are similar, namely $\approx 50 \%$ and $70 \%$, respectively. Whereas the former, dominant component can be expected to be close to spherical, the latter will show the imprint of the explosion. In contrast, $\mathrm{Si} / \mathrm{Ca}$ are mostly produced during the explosion and reflect the explosion properties. As discussed by Hoeflich et al. (2006) within the framework of off-centered delayed detonation models developed for SN 2004dt, O shows no (or very weak) polarization because the pre-explosion $\mathrm{O}$ is already sufficient to block all direct light emitted from the photo-disk (the photosphere cross section along the line of sight). This means there is no large scale, chemical asymmetry at the wavelengths corresponding to the blue-shifted O absorption.

Besides the Si II $6355 \AA$ and the near-IR Ca II triplet, the only spectral features for which polarization is detected at a significant level are Si II $5051 \AA$ and, less markedly, Mg II $4471 \AA$ (see Figs. A.1-A.7). Figure 24 illustrates the evolution of Si II $5051 \AA$. While on days -10 and -8 the signalto-noise is too low, on day -6 the polarization of this line is detected at the $3-\sigma$ level $(0.37 \pm 0.11 \%)$. On day -1 the feature is still there $(0.36 \pm 0.06 \%)$, while by day +39 it has completely disappeared below the detection limit $(\leq 0.2 \%)$. In general, the polarization peak appears to be broader than that of Si II $6355 \AA$ (see Fig. 12) and this might be due to the presence of some other lines, whose absorption troughs are clearly visible in the flux spectrum (Fig. 24). Less clear is the case of Mg II 4471 Å. Even though a polarized feature is consistently present from day -8 on, the detection level is significant only in the epochs immediately preceding maximum light, when the polarization reaches 


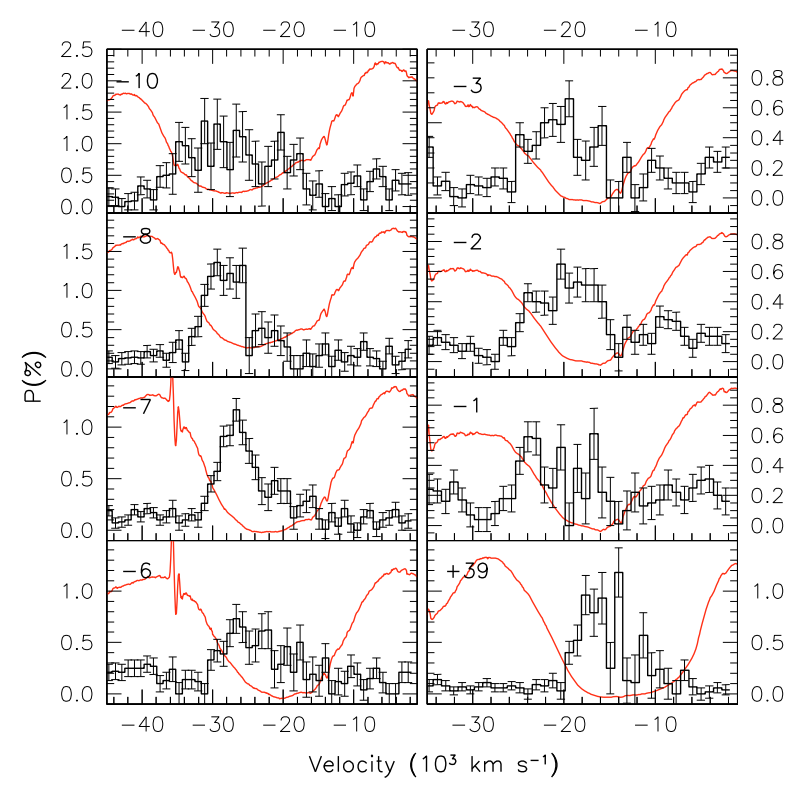

Fig. 16. Polarimetric evolution of the Ca II IR triplet profile. The smooth solid curve traces the unbinned flux spectrum, arbitrarily scaled for presentation. Note the different vertical and horizontal scales in each plot.

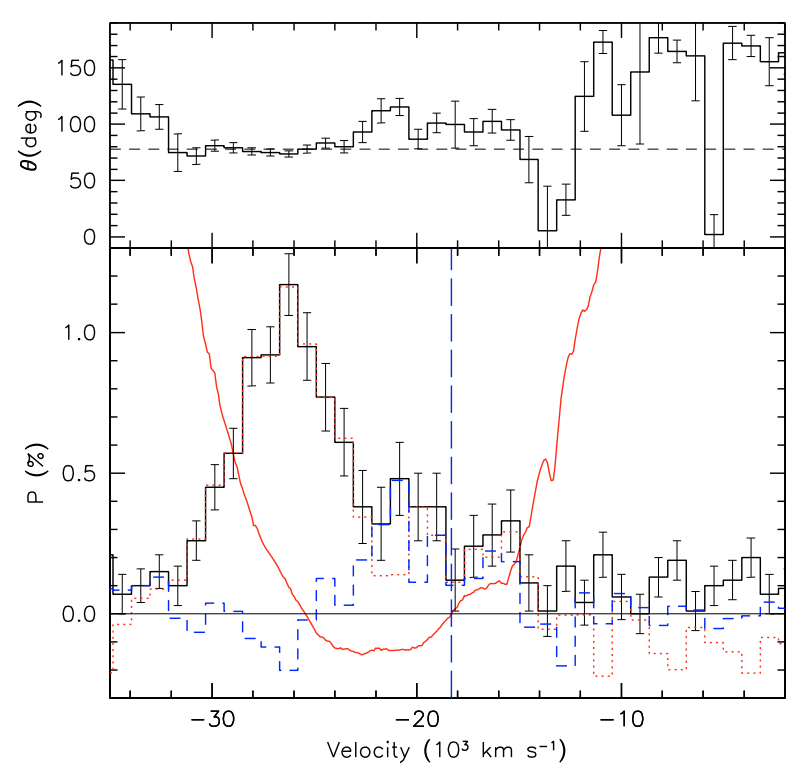

Fig. 17. Lower panel: polarization profile of Ca II IR triplet on day -7 . Dotted and dashed lines trace $P_{d}$ and $P_{0}$ respectively (see Sect. 5.1). The vertical long-dashed line indicates the expansion velocity deduced from the Si II $6355 \AA$ Aline at the same epoch. Upper panel: polarization position angle. The horizontal dashed line indicates the average angle computed in the velocity range $-30000 \leq v \leq-24000 \mathrm{~km} \mathrm{~s}^{-1}$. The feature around $-15000 \mathrm{~km} \mathrm{~s}^{-1}$ is due to a telluric absorption.

$0.5 \pm 0.15 \%$ (see Figs. A.10, A.11). In general, the $Q-U$ diagrams for these two weak features are quite noisy and it is very difficult to say whether a dominant axis exists or not.

\subsection{The $6400-7200 \AA$ continuum}

The region between 6400 and $7200 \AA$ is practically free of strong features (Kasen et al. 2004; Leonard et al. 2005; Wang et al. 2006b) and can therefore be used to estimate the continuum polarization. In the case of SN 2006X, the average polarization

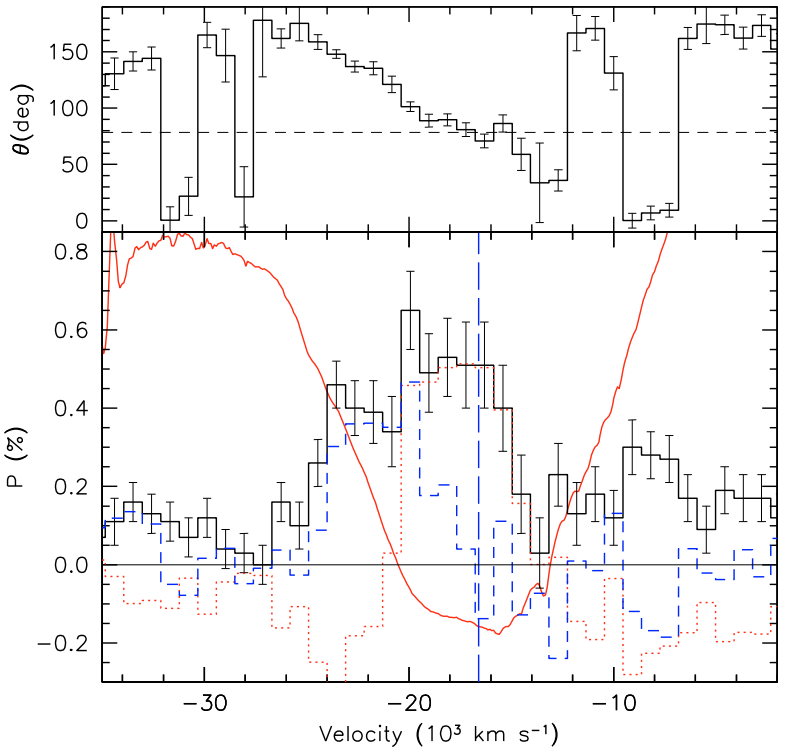

Fig. 18. Same as Fig. 17 for day -2 . The horizontal dashed line in the upper panel indicates the orientation of the dominant axis deduced on day -7 .

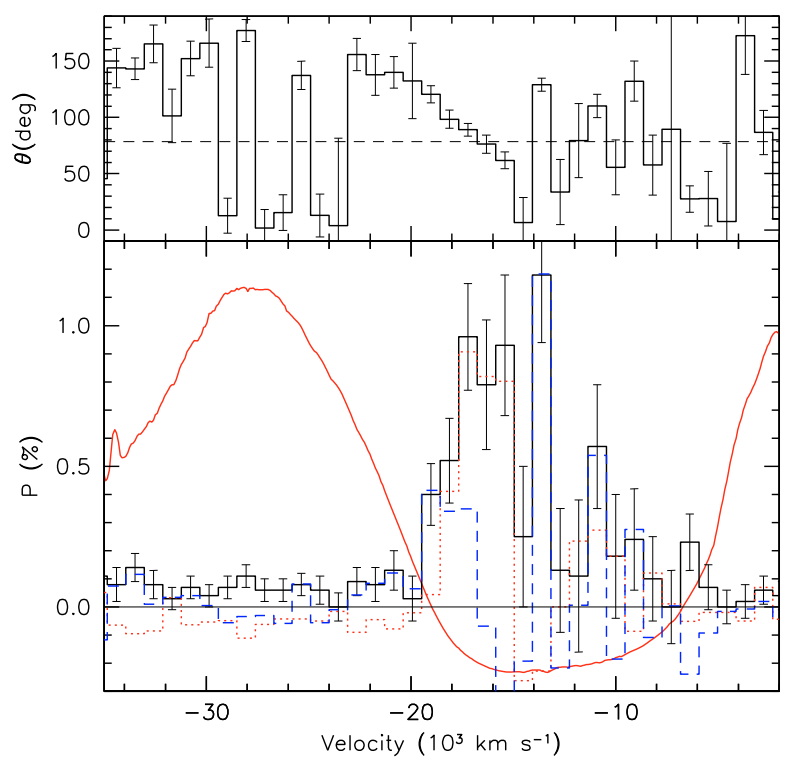

Fig. 19. Same as Fig. 17 for day +39 . The horizontal dashed line in the upper panel indicates the dominant axis deduced on day -7 .

level computed in this spectral range is below $0.2 \%$ at all epochs, which is consistent with a very small departure from spherical symmetry in the photosphere ( $\leq 10 \%$; Höflich 1991), even though fluctuations at the level of $3-4 \sigma$ from the average value are seen (see Fig. 25). Finally, the analysis of the $Q-U$ plane for the data in the wavelength range $6400 \AA \leq \lambda \leq 7000 \AA$ does not show any significant dominant axis. Incidentally, this confirms that the ISP was properly removed by the procedure outlined in Sect. 4.

\section{Discussion and conclusions}

SN 2006X is characterized by a number of distinguishing features: a) strong reddening and peculiar extinction law; b) extremely high expansion velocities; c) intense high-velocity Ca II 

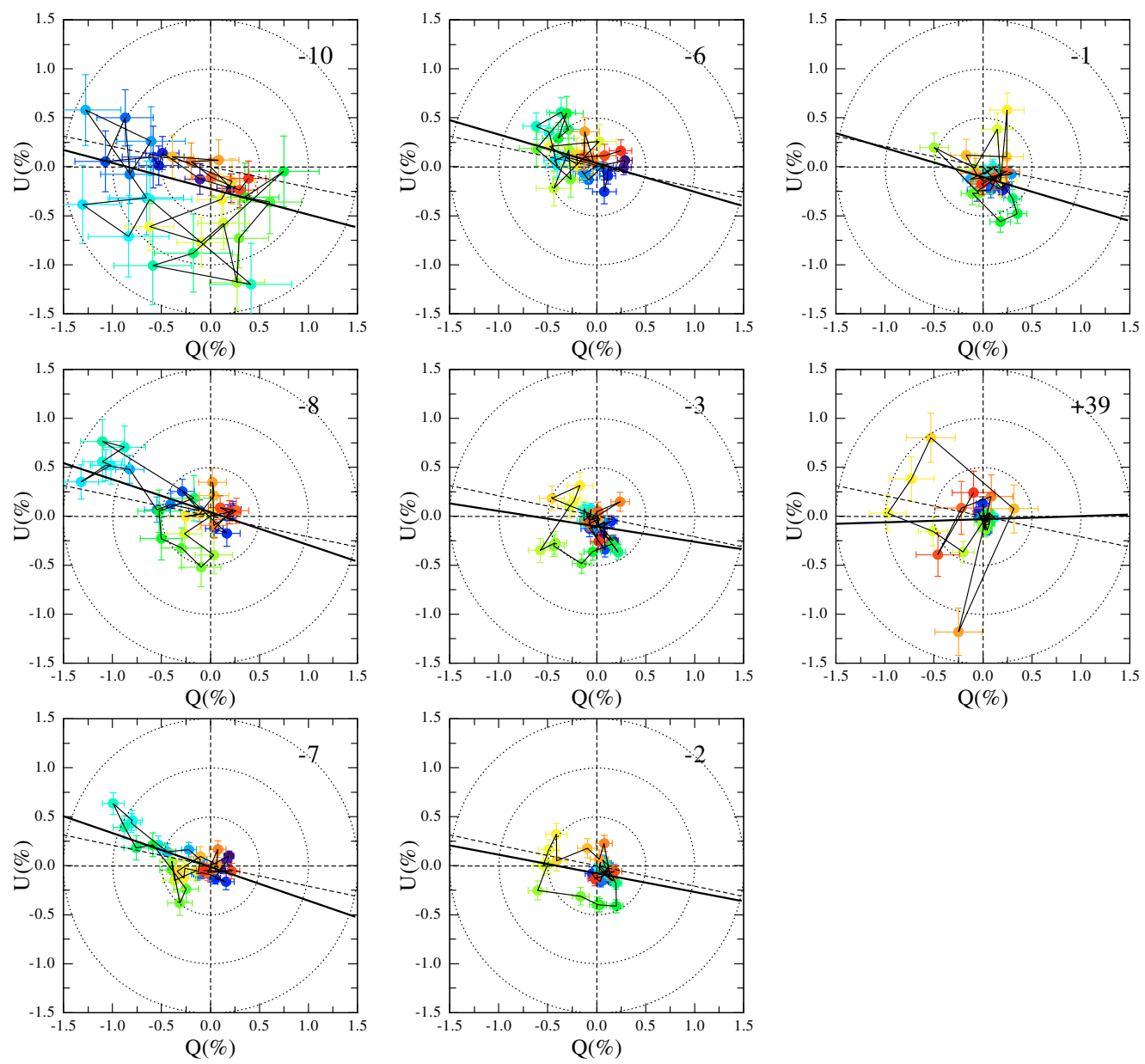

$\mathrm{Q}(\%)$

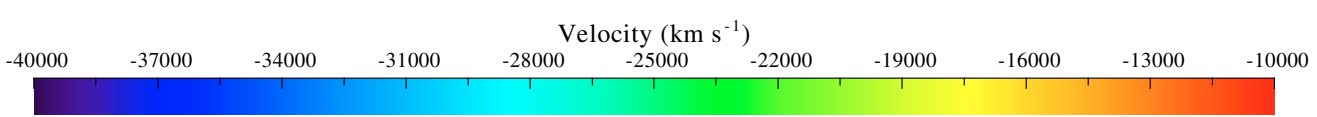

Fig. 20. $Q-U$ diagrams for the Ca II IR triplet (see Fig. 15). The velocity scale is computed with respect to the average triplet wavelength (8579.1 ̊).

features; d) possible detection of circumstellar material; e) detection of a light echo. These facts have led Wang et al. (2008a) to propose that SN 2006X and other rapidly expanding objects (like SN 2002bo) belong to a subclass with distinct photometric and spectroscopic properties, possibly associated with dusty environments. A similar proposal was put forward for SN 2004dt by Wang et al. (2006b), who suggested that these events might come from "younger" progenitor stars.

Also from a spectro-polarimetric point of view SN 2006X shows a number of distinguishing features, each of them deserving a separate discussion.

\subsection{General spectropolarimetric properties}

Besides SN 2001el, SN 2006X is the only type Ia SN with a very good spectropolarimetric coverage during the pre-maximum phase and with data extending up to more than one month past maximum. This has allowed us to study the variations shown by the most prominent features. In particular, the polarization of the Si II $6355 \AA$ line appears to follow the trend displayed by the fast expanding SN 2002bo (Wang et al. 2007), as shown in Fig. 26. In a scenario where the polarization across the absorption profiles is generated by clumps each of them having a significant photospheric covering factor (Wang et al. 2003; Kasen et al. 2003), the observed fluctuations might be explained in terms of changes in the covering factor itself as the photosphere recedes in velocity with time. Nevertheless, given the relatively large errorbars, it is not possible to deduce a systematic physical difference between the two events (e.g. different extension and number of clumps in the ejecta of the two objects). Only future observations with higher accuracy will enable this kind of analysis for newly discovered, nearby SNe.

As shown by Wang et al. (2007), there is an anti-correlation between the luminosity of type Ia $\mathrm{SNe}$ and their degree of asphericity. In order to properly place our object into the Wang et al. (2007) plot, we have estimated the Si II $6355 \AA$ polarization degree $P_{\text {SiII }}$ on day -5 using a second order polynomial fitting to the observed data (1.0\%; see Fig. 26, dashed curve). As for the error-bar, we have adopted the same value estimated for the observed points at comparable epochs $(0.1 \%)$. Once this is done, SN 2006X conforms to the behavior shown by the other SN Ia, as we show in Fig. 27 (after excluding the two most deviating SNe 1999by and 2004dt the Pearson correlation coefficient is 0.85 , and the rms deviation of the data points from the best fit is $0.14 \%$ ). Interestingly, such a relation had been predicted 


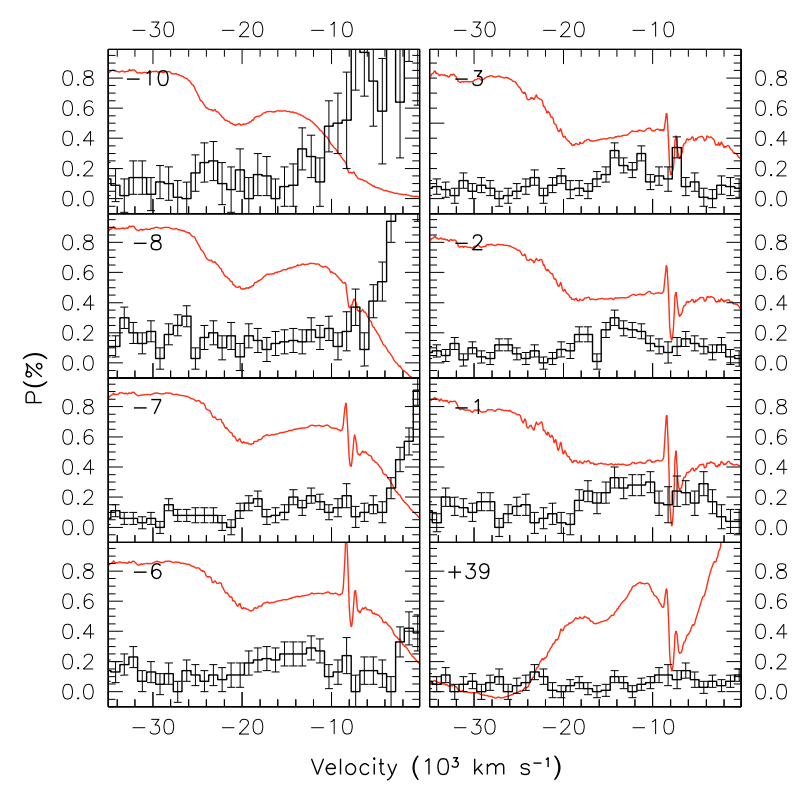

Fig. 21. Polarimetric evolution of the O I $7774 \AA$ line profile. The smooth solid curve traces the unbinned flux spectrum, arbitrarily scaled for presentation. The broad polarized feature on the right edge is the blue wing of the $\mathrm{Ca}$ II near-IR triplet.

within the delayed detonation models, where initial chemical inhomogeneities, in the form of $\mathrm{Ni}$ clumps in the $\mathrm{Si} / \mathrm{O}$ rich outer layers, are expected to be produced during the deflagration phase (Khokhlov 1995; Lisewski et al. 2000; Gamezo et al. 2003). However, the subsequent detonation will burn most of the intermediate mass elements to ${ }^{56} \mathrm{Ni}$ and, thus, eliminate most of the chemical inhomogeneities at an efficiency which increases with the total production of ${ }^{56} \mathrm{Ni}$, i.e. with the luminosity (Khokhlov 2001; Höflich et al. 2002; Wang et al. 2007; Mazzali et al. 2007; Kasen et al. 2009).

SN 2006X shows one of the highest polarizations ever observed in a Ia and it lies at the upper edge of the Wang et al. (2007) relation. As in all other known cases, the Si II polarization disappears after maximum light (see Fig. 12).

The line polarizations discussed here can be understood in a simple topological picture, which has been confirmed by detailed models. Thomson scattering produces null polarization in the case of forward scattering, while the polarization is maximum if the photons are scattered by 90 degrees. Consequently, the resulting polarization of an individual element of the photodisk increases with its distance from the center. Polarization by lines can be understood as follows: a line "blocks out" at certain wavelengths the (polarized) flux from the Thomson scattering dominated photo-disk. If the amount of blocking is not distributed evenly around the line of sight, the result is that the integrated polarization over the entire disk is not null (Wang et al. 2003; Kasen et al. 2003; Wang et al. 2006b; Höflich et al. 2006). Uneven blocking/optical depths can be caused by asymmetric chemical and/or density distributions.

At early times, the Si II optical depth is high above some parts of the Thomson scattering photo-disk but low at others and this asymmetric "shadowing" turns into a net polarization. As the photosphere shrinks with time, an increasingly higher velocity/frequency range can be blocked, because the $\mathrm{Si}$ abundance remains high over a large velocity range. Eventually, for every point of the photo-disk, there is a region in the atmosphere where

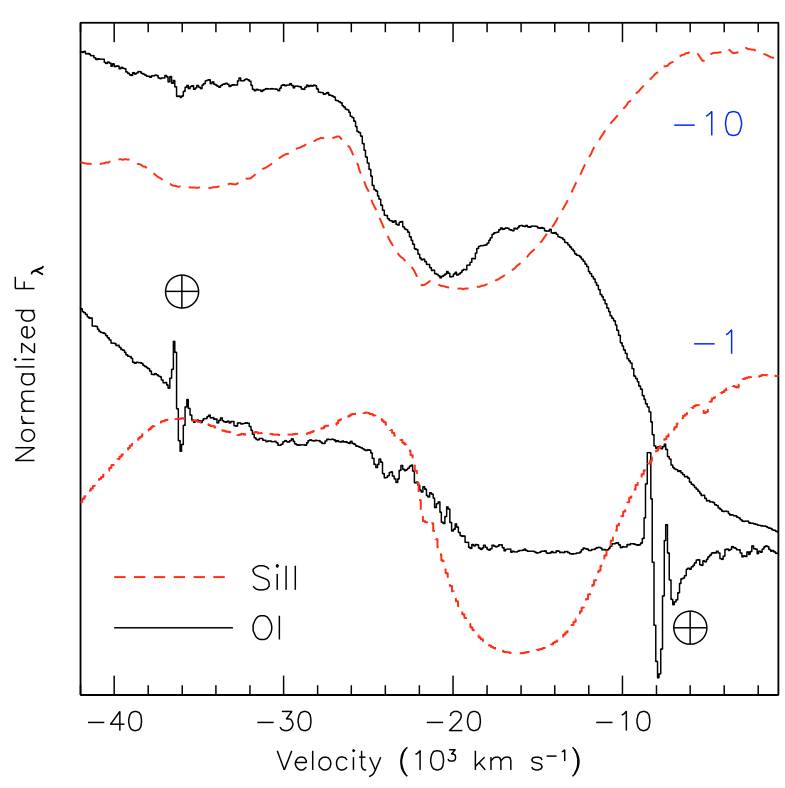

Fig. 22. O I $7774 \AA$ A and Si II $6355 \AA$ A line profiles on days -10 (top) and -1 (bottom).

the Si II line blocks the direct emission from the photosphere and, thus, the polarization vanishes.

For the Si II line, there is another effect, which is equally important and goes in the same direction. The Sobolev optical depth of Si II is about 30 to 80 at the photosphere (Höflich et al. 1993; Höflich 1995), but the chemically asymmetric outer layers become optically thin due to geometrical dilution. This is not the case for the Ca II absorption at several weeks after maximum light, since the outer calcium layers remain optically thick because the line opacity is larger by several orders of magnitude.

The strong polarization shown by $\mathrm{Si}$ II and $\mathrm{Ca}$ II, as opposed to the lack of detection for O I despite the similar position in velocity space, associates SN 2006X to SN 2004dt and leads us to conclude that, as in that case, the distribution of oxygen is roughly spherically symmetric but is contaminated by intermediate-mass elements extending into the oxygen-rich region (Wang et al. 2006b). In this respect, we note that an offcenter delayed-detonation model reproduced rather well the high polarization and polarization angle of $\mathrm{Si}$ and $\mathrm{Ca}$ and the low polarization of O during the early stages of SN 2004dt (Höflich et al. 2006).

Another feature common to SN 2004dt and SN 2006X is the very high expansion velocities shown by both $\mathrm{SNe}$. Nevertheless, other properties make SN 2006X more closely resemble SN 2001el (Wang et al. 2003). Both SN 2006X and SN 2001el share a larger intensity of the Ca II HVF and its distinct polarization parameters with respect to the photospheric component. At the time of SN 2001el, the recognition of HVFs was only emerging. Nevertheless, Wang et al. (2003) commented that if one of the explanations discussed by them, namely a toruslike structure, were right, occurrences of strong HVFs should be strongly polarized (a low number of large clumps would have a similar effect. See also Kasen et al. 2003 and Tanaka et al. 2006 for the effects of toroidal distributions of material). SN 2004dt has shown that the opposite is not (always) true: this supernova's Ca II HVF was highly polarized whereas the line strength was lower than in both SN 2001el and SN 2006X. In SN 2006X, the Ca II IR HVF is strong in both equivalent width and polarization, thereby matching SN 2001el. But, unlike SN 2001el, the 


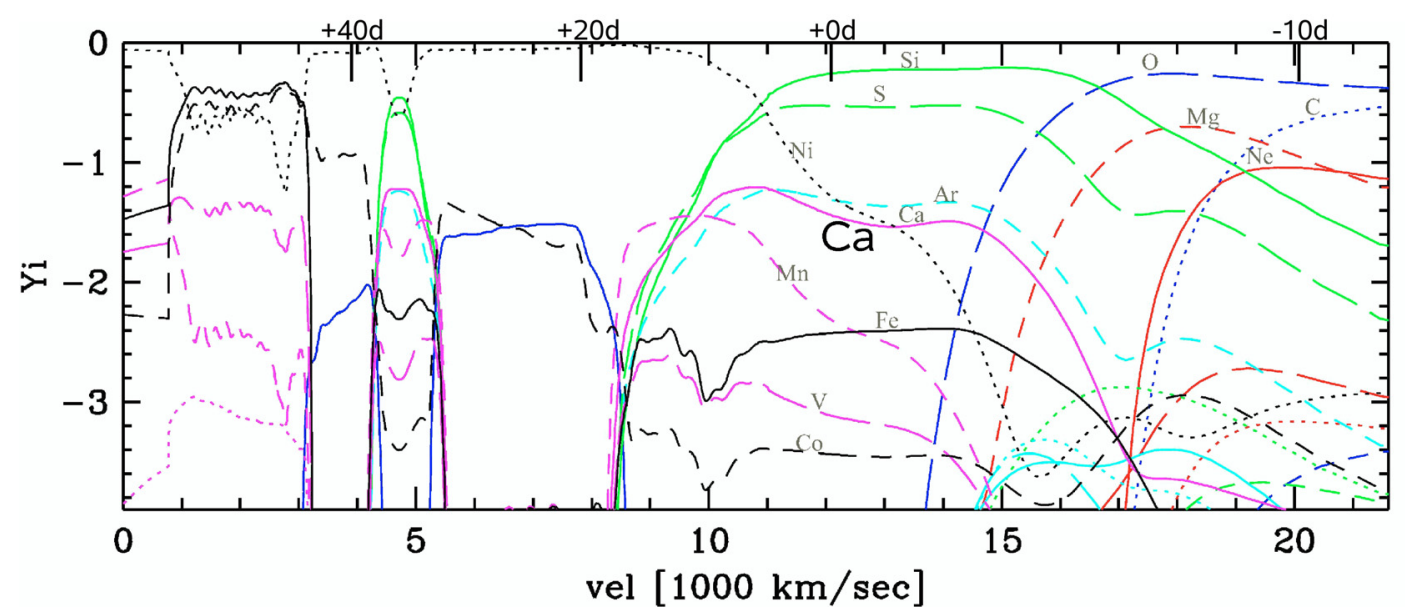

Fig. 23. Abundances as a function of expansion velocity for a delayed detonation model of a $M_{V}=-19.29$, spectroscopically normal SN Ia originating from a star with a main sequence mass of $5 M_{\odot}$. The deflagration/detonation transition density is $2.5 \times 10^{7} \mathrm{~g} \mathrm{~cm}^{-3}$ (Höflich et al. 2002). For all but very early times, $\mathrm{Ca}$ and $\mathrm{Si}$ are singly ionized. Tickmarks on the upper abscissa mark the velocities of the Thomson-scattering photosphere at various times relative to maximum light. Within this series of delayed-detonation models for spectroscopically normal SNe Ia, $M_{V}$ is expected to range between -18.98 and -19.35 , and expansion velocities vary by about $15 \%$.

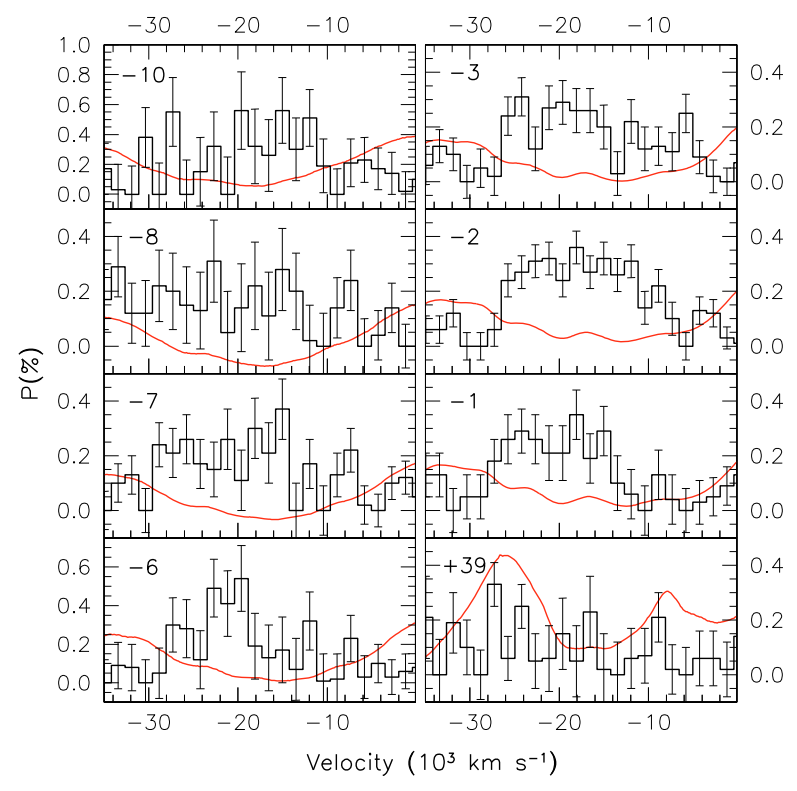

Fig. 24. Polarimetric evolution of the Si II $5051 \AA$ line profile. The smooth solid curve traces the unbinned flux spectrum, arbitrarily scaled for presentation.

polarization angle of the HVF is not very different from the one of the dominant axis.

\subsection{The near IR Ca II triplet and its HVF}

One of the most interesting aspects shown by the polarization data of SN 2006X is the behavior of the Ca II near-IR triplet and the distinct polarization properties of the HV component with respect to the photospheric component (see Figs. 17-19). In general, we believe this is consistent with the Ca II line-forming regions discussed by Kasen et al. (2003) and Gerardy et al. (2004). If the HV component is formed in a region that asymmetrically shadows the Thomson scattering photosphere, it will lead to an incomplete cancellation of the photospheric polarized light. In turn, this can explain the loop on the $Q-U$ plane displayed by

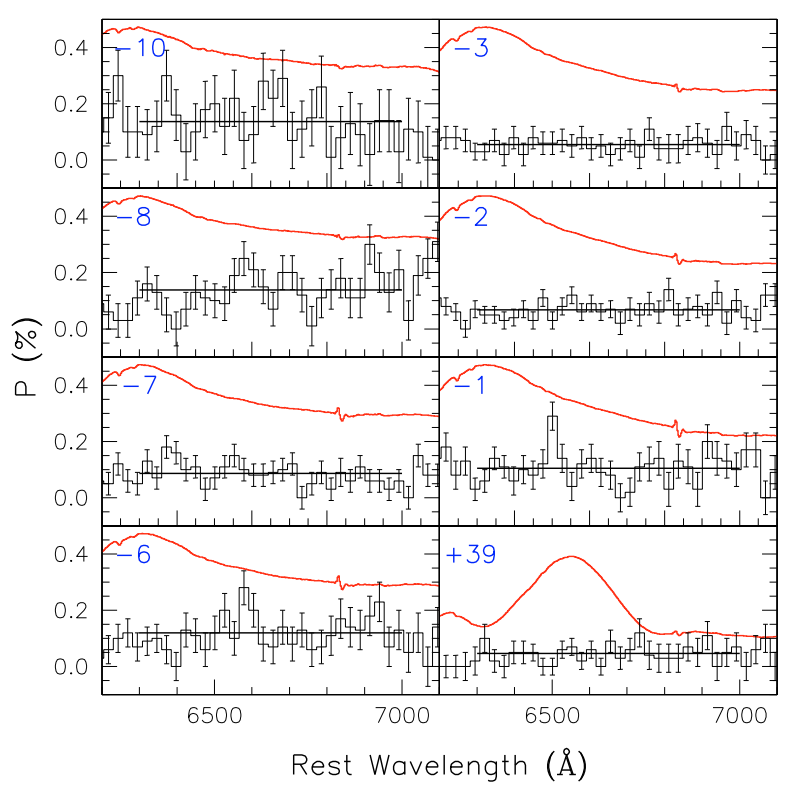

Fig. 25. Polarimetric evolution of the $6200-7000 \AA$ continuum. The smooth solid curve traces the unbinned flux spectrum, arbitrarily scaled for presentation. The horizontal line is placed at the average polarization level within the wavelength range 6300-7000 $\AA$.

the Ca II HV component in Fig. 20 (Kasen et al. 2003; Wang \& Wheeler 2008). Moreover, blending the more polarized HV and less polarized photospheric components (as is the case for SN 2006X) also accounts for the observed blue-shift of the polarization peak with respect to the line center in the flux spectrum.

Clearly, the time evolution shown by the two components indicates a scenario which is more complicated than that derived for SN 2001el, for which the Ca II photospheric component did not show a significant polarization (Wang et al. 2003; Kasen et al. 2003). Also at variance with the case of SN 2001el, SN 2006X shows a comparable polarization for the Si II $6355 \AA$ line, and it displays a loop in the $Q-U$ plane prior to maximum light (see Fig. 15), indicating that this feature is 


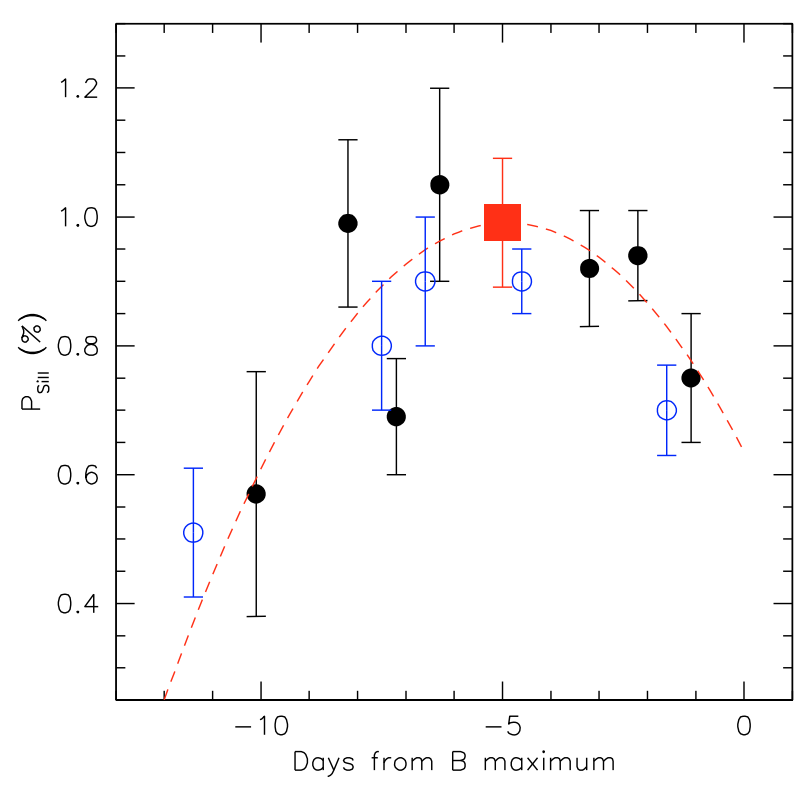

Fig. 26. Linear polarization of Si II $6355 \AA$ as a function of epoch. The dashed line is a second order polynomial fitting to SN 2006X data and the filled square dot is the polarization interpolated for day -5 (see Sect. 6.1). The empty circles are the data for SN 2002bo (Wang et al. 2007).

formed within an odd shaped line-forming region, at least in the early epochs. Moreover, the Si II and Ca II loops tend to lie in the $-Q,+U$ quadrant of the $Q-U$ plane (Figs. 15, 20).

This might imply that there is an extended protrusion of peculiar shape, exterior to the photosphere and photospheric lineforming regions, that gives rise to the Ca II HV component and, to a lesser extent, some of the Si II feature. It is rather intriguing that the HV Ca II and the photospheric Si II features appear to share the same dominant axis, at least in the earliest epochs (compare Fig. 13 to Fig. 17).

Even though the Ca II HVFs are a common feature in the early, pre-maximum spectra of type Ia SNe (Mazzali et al. 2005a), their origin is still debated. The most favored scenario is one where these features arise within circumstellar material that is overrun by the rapidly expanding outermost layers of the SN ejecta (Gerardy et al. 2004). An additional phenomenon is the $\mathrm{Ca}$ (and possibly $\mathrm{Si}$ ) abundance enhancement in the outer regions of the explosion (Mazzali et al. 2005a), which however requires also density enhancements (i.e. a 3D structure in the explosion) in order to account for the strength of the HVF (Mazzali et al. 2005b). Quimby et al. (2006b) point out that the CSM interaction picture is supported by the velocity cutoff seen in the Si II $6355 \AA$ blue wing profile. In the case of SN 2006X, our data show that the HVF is blended with the photospheric feature indicating, in the scenario proposed by Gerardy et al. (2004), a high mass for the shell produced by the interaction (for SN 2003du Gerardy et al. estimated a mass of $\approx 10^{-2} M_{\odot}$; see also Mazzali et al. 2005a; and Tanaka et al. 2006). This blending would also be consistent with the Si II velocity cutoff and the velocity shift between the HVF and the photospheric component (Gerardy et al. 2004; Quimby et al. 2006a) which, on day -10, is about $9000 \mathrm{~km} \mathrm{~s}^{-1}$ (Fig. 5).

We notice that this value is higher than the $\sim 7000 \mathrm{~km} \mathrm{~s}^{-1}$ seen in 2002bo (Mazzali et al. 2005a) and 2004du (Gerardy et al. 2004). Shell masses apparently vary in a wide range (e.g. $\approx 5 \times 10^{-3} M_{\odot}$ for SN $2005 \mathrm{cg}$, Quimby et al. 2006a, to $5 \times 10^{-2} M_{\odot}$ for SN 2002bo, Gerardy et al. 2004) and, consequently, the cutoff velocity is also expected to do so. Whether this is related to the extremely high velocities recorded for SN 2006X or is rather due to some other effect, remains to be clarified.

Normally the HVF are well visible in the very early stages of the SN evolution and disappear around maximum light (Mazzali et al. 2005a). The spectropolarimetry shows a clear component at about $-25000 \mathrm{~km} \mathrm{~s}^{-1}$ on day -1 , to be compared with the $-16300 \mathrm{~km} \mathrm{~s}^{-1}$ deduced from the minimum of the Si II $6355 \AA$ absorption trough. From this we deduce that the HVF component is still present around maximum light, and its blue-shift has decreased by about $2000 \mathrm{~km} \mathrm{~s}^{-1}$ from day -8 . We notice that this variation is not very different from the one shown by the expansion velocity in the same time interval $\left(\sim 2500 \mathrm{~km} \mathrm{~s}^{-1}\right.$, see Fig. 3).

If the HV Ca feature is indeed produced by the interaction between the SN ejecta and the surrounding "non-processed" material, then the polarization observed across the HVF implies that this material is distributed in an asymmetric way.

\subsection{Ca II re-polarization}

The most striking event in the evolution of SN 2006X is the polarization observed on day +39 , peaking at about $-16000 \mathrm{~km} \mathrm{~s}^{-1}$ (Fig. 19). The absorption profile is rather broad for this relatively late epoch: it is broader than on day -1 (Fig. 16) and in SN 2002bo at a comparable epoch (Fig. 4). The difference with SN 2002bo is produced by the strong component at $-16000 \mathrm{~km} \mathrm{~s}^{-1}$ that gives rise to a marked line profile asymmetry.

The expansion velocity deduced from the Si II $6355 \AA$ for day +30 is about $12000 \mathrm{~km} \mathrm{~s}^{-1}$ (Wang et al. 2008a), while the minimum of the absorption trough in our last spectrum is $\sim 13000 \mathrm{~km} \mathrm{~s}^{-1}$. A lower value $\left(\sim 9500 \mathrm{~km} \mathrm{~s}^{-1}\right)$ is deduced by interpolating the Fe II $4555 \AA$ line velocities reported by Wang et al. (2008a) to day +40 . Strong lines are formed well above the photosphere and, if the abundance of a specific element declines, then the expansion velocity does not follow the receding photosphere but levels off in velocity space as in the case of Si II (e.g. Höflich \& Khokhlov 1996; Benetti et al. 2004). The fact that on the same late epoch there is no trace of Si II $6355 \AA$ line polarization (Fig. 12) indicates that there must be a substantial difference in optical depth and geometry between the regions where calcium and silicon lines are generated in this phase.

The disappearance of the polarization in the Si features can be understood in the framework of off-center DDT models. Early on, the $\mathrm{Si}$ lines are formed in a region where, for a given radius, the Si abundance is direction dependent. This causes an "asymmetric" covering of the photosphere (see Fig. 2 in Höflich 2006) leading to a net polarization. As time goes by, the photosphere and the Si line forming region recede well within the Si-rich region, so that there is no selective "covering" of the photosphere and hence no Si polarization. For this reason the Si polarization disappearance is a general phenomenon, expected to take place in any scenario/model with an extended, aspherical region of incomplete burning $(\mathrm{Si} / \mathrm{S})$. Therefore, it is not limited to off-center DDT or DD models, provided the SN undergoes a detonation phase with O-burning.

At our last epoch, about 40 days past maximum light, the photosphere has receded below $5000 \mathrm{~km} \mathrm{~s}^{-1}$, well into the ${ }^{56} \mathrm{Ni}$-rich region (Fig. 23), where $\mathrm{Ca}$ is a product of the explosive nucleosynthesis and its abundance is about 1 to $2 \%$, i.e. enhanced by a factor of $\approx 10^{4}$ with respect to its solar value. 


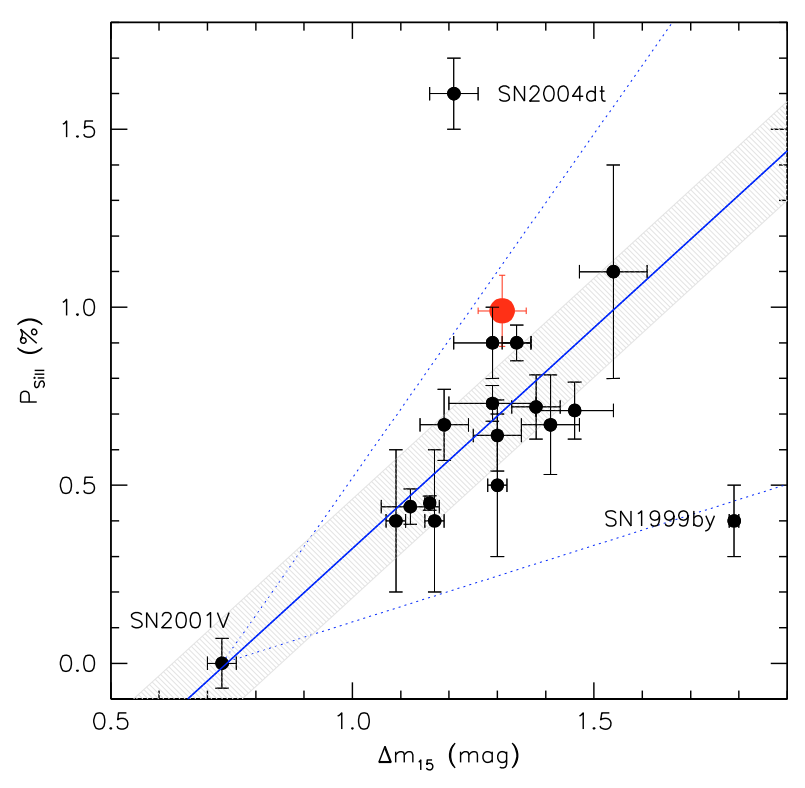

Fig. 27. Degree of polarization across the Si II $6355 \AA$ line as a function of light curve decline rate. Data are from Wang et al. (2007). SN 2006X is marked by the large dot. The solid line is a best fit to all data, with the exception of SN 1999by and SN 2004dt. The dotted lines trace the 1- $\sigma$ level of the intrinsic polarization distribution generated by the Monte-Carlo simulation discussed by Wang et al. (2007). The shaded area indicates the rms deviation of the data points from the best fit relation.

For a spectroscopically normal SN Ia (Hoeflich et al. 2002), a Ca-rich region of almost constant abundance is predicted between 9000 to $15000 \mathrm{~km} \mathrm{~s}^{-1}$ (Fig. 23) ${ }^{3}$. The abundance of Ca declines logarithmically and smoothly outward, converging to a solar value between 17500 to $19000 \mathrm{~km} \mathrm{~s}^{-1}$. This is particularly interesting, because the outer edge (placed at about $15000-17000 \mathrm{~km} \mathrm{~s}^{-1}$; see Fig. 23) coincides with the velocity at which we see strong polarization several weeks after maximum (Fig. 19), when the scattering-dominated photosphere is receding through the ${ }^{56} \mathrm{Ni}$ region.

Note that theoretical spectra do not show a significant polarization in the photospheric component of Ca II during the early times when the photosphere recedes from 17000 to $12000 \mathrm{~km} \mathrm{~s}^{-1}$ (Höflich et al. 2006) because of the rather smooth decline in abundance and the huge cross section of the Ca-IR triplet which causes the line to be optically thick $(\tau \approx 1)$, even for abundances close to solar (Fig. 23). The Ca II lines cover up the entire photosphere, leading to a low polarization of the photospheric component similar to that displayed by oxygen, although the asymmetries in $\mathrm{Ca}$ caused by off-center delayed detonation (see the discussion in Sect. 5.3) are similar in size to those of the Si-rich layers (Fesen et al. 2007). Similarly to the behavior of $\mathrm{Si}$, the polarization is low when the photosphere is well within the Ca-rich layers because of the large extension of $\mathrm{Ca}$ in velocity space $\left(\approx 8000\right.$ to $17000 \mathrm{~km} \mathrm{~s}^{-1}$, see Fig. 23 ; see also Branch et al. 2006) and because almost all of the inner, Thomson scattering dominated atmosphere is covered up by $\mathrm{Ca}$ at a range of expansion velocities.

Several weeks after maximum cooling and geometric dilution cause the optical depth to decrease by more than an order

3 The Ca-rich layer seen in Fig. 23 at about $5000 \mathrm{~km} \mathrm{~s}^{-1}$ is an artifact of spherically symmetric delayed-detonation models and is related to the fact that the initiation of the delayed-detonation is located in a shell (Khokhlov 1995; Höflich 1995). of magnitude at the outer boundary of the Ca-rich region. By that time, the photosphere has receded within the inner edge of the $\mathrm{Ca}$ layer at $8000-9000 \mathrm{~km} \mathrm{~s}^{-1}$. The result is a partial cover up of the photosphere in the line wings that generate a polarization comparable to the one shown by $\mathrm{Si}$, because of the similar size of asymmetry of the $\mathrm{Si}$ and $\mathrm{Ca}$ layers. The large extension of $\mathrm{Ca}$ in velocity space $\left(\Delta v=10000 \mathrm{~km} \mathrm{~s}^{-1}\right)$ is consistent with its observed broad absorption. It is expected that a large fraction of the inner Thomson scattering region $\left(v_{\mathrm{ph}} \approx 4000 \mathrm{~km} \mathrm{~s}^{-1}\right)$ is covered-up by $\mathrm{Ca}$ at most wavelengths. In the line wings where the Sobolev optical depth of the line drops to a few, the blocking is less, resulting in high polarization. The fact that we do not see a statistically significant polarization at the inner $\mathrm{Ca}$ boundary $\left(v \lesssim 10000 \mathrm{~km} \mathrm{~s}^{-1}\right.$; see Fig. 19) is a probable sign of mixing at the inner edge of the Ca-rich layers (8000 $\mathrm{km} \mathrm{s}^{-1}$, see Fig. 23).

Unfortunately, a single observation does not provide information separately for the size of the asymmetry, the orientation of the observer and mixing processes which are expected to "soften" the steep gradients in layers with rapid changes of abundances. It is the time dependence, which is direction dependent for a given supernova, that tells us how fast the photosphere passes a given chemical "boundary layer". This is why it will be very important, in the future, to follow type Ia SNe with multiepoch spectropolarimetry at epochs later than a month. This will allow us to understand whether the re-polarization is a transient phenomenon (as it should be) and tell us something about mixing. In particular, the mixing component should introduce a change of the dominant axis with time on short time-scales, whereas large scale asymmetries should cause slow variations. Time coverage would thus allow us to separate the different components. In this respect, for the case of SN 2006X, the data are inconclusive. While a dominant axis is present before maximum light (Figs. 17, 18), it is rather unclear at the last epoch. There might be an indication that the $-16000 \mathrm{~km} \mathrm{~s}^{-1}$ component has a polarization angle similar to the one displayed by the feature at a similar velocity just before maximum light (Fig. 19), but we do not attach too much confidence to this fact.

Because basically all observations have shown $\mathrm{SNe}$ Ia to become unpolarized within a week after $B$ maximum light, observational series have routinely been discontinued shortly after peak brightness; but 2 out of 2 SNe Ia observed at considerably later phases (SN 2006X and SN 2001el) now show convincingly the tomographic power given by the combination of spectroscopy and polarimetry. The case of SN 2006X demonstrates that spectropolarimetry enables one to probe regions which are dominated by the early phases of the deflagration burning and the thermonuclear runaway. Late time polarization, both in size and timing, will depend on the viewing angle and on a variety of other aspects. Among these are the location at which the deflagration front turns into a detonation and the amount of mixing produced by instabilities during the deflagration phase (plumes), which may wash out abundance gradients by different amounts (Fesen et al. 2007; Hoeflich et al. 2002).

While SN 2001el did not show polarization at late epochs, SN 2006X certainly did. This may be due to the quality and timing of observations, orientation effects or may hint at an intrinsic diversity, thus providing a glimpse of what we might learn in the future by combining extensive spectropolarimetric coverage with systematic theoretical studies.

Acknowledgements. This paper is based on observations made with ESO Telescopes at Paranal Observatory under program IDs 076.D-0177(A) and 076.D-0178(A). The authors are grateful to ESO-Paranal staff for the support given during the service mode. This work is partially based on NSF grants AST 04-06740,07-03902 \& 07-08855 to P.A.H., AST-0707769 to J.C.W. and 
AST-0708873 to L.W. F.P. wishes to thank Dr P. Danielson for her kind help. The authors are grateful to an anonymous referee for her useful comments.

\section{References}

Appenzeller, I., Fricke, K., Fürtig, W., et al. 1998, The Messenger, 94, 1 Arnett, W. D. 1969, ApJ, 157,1369

Benetti, S. 1989, Master Thesis, Padua University

Benetti, S., Meikle, P., Stehle, M., et al. 2004, MNRAS, 348, 261

Benetti, S., Meikle, P., Stehle, M., et al. 2005, ApJ, 623, 1011

Branch, D., Dang, L. C., Hall, N., et al. 2006, PASP, 118, 560

Branch, D., Jeffery, D. J., Parrent, J., et al. 2008, PASP, 120, 135

Chornock, R., \& Filippenko, A. V. 2007, AJ, 136, 2227

Chugai, N. N. 2008, Astron. Lett., 34, 389

Cox, N., \& Patat, F. 2008, A\&A, 485, L9

Cropper, M., Bailey, J., McCowage, J., Cannon, R. D., \& Couch, W. J. 1988, MNRAS, 231, 695

Crotts, A., \& Yourdon, D. 2008, ApJ, 689, 1186

Crutcher, R. M. 1985, ApJ, 288, 604

Ferrarese, L., Freedman, W. L., Hill, R. J., et al. 1996, ApJ, 464, 568

Fesen, R. A., Höflich, P. A., Hamilton, A. J. S., et al. 2007, ApJ, 658, 396

Gamezo, V. N., Khokhlov, A. M., Oran, E. S., et al. 2003, Science, 299, 77

Gerardy, C.L., et al. 2004, ApJ, 207, 391

Hachinger, S., Mazzali, P. A., \& Benetti, S. 2006, MNRAS, 370, 299

Höflich, P. 1991, A\&A, 246, 481

Höflich, P. 1995, ApJ, 443, 89

Höflich, P., \& Khokhlov, A. 1996, ApJ, 457, 500

Höflich, P., Müller, E., \& Khokhlov, A. 1993, A\&A, 268, 570

Höflich, P., Gerardy, C. L., Fesen, R. A., \& Sakai, S. 2002, ApJ, 568, 791

Höflich, P., Gerardy, C. L., Marion, G. H., \& Quimby, R. 2006, New Astron. Rev., 50, 470

Howell, D. A., Höflich, P., Wang, L., \& Wheeler, J. C. 2001, ApJ, 556, 302

Immler, S. I., Brown, P. J., Milne, P., et al. 2006, ApJ, 648, L119

Kasen, D., Nugent, P., Wang, L., et al. 2003, ApJ, 593, 788

Kasen, D., Nugent, P., Thomas, R. C., \& Wang, L. 2004, ApJ, 610, 876

Kasen, D., Röpke, F. K., \& Woosley, S. E. 2009, Nature, 460, 869

Khokhlov, A. 1995, ApJ, 449, 695

Khokhlov, A. 2001, ApJ, submitted [arXiv: astro-ph/0008463]

Khokhlov, A., Müller, E., \& Höflich, P. 1993, A\&A, 270, 223

Lauroesch, J. T., Crotts, A. P. S., Meiring, J., et al. 2006, CBET, 421

Leonard, D. C., \& Filippenko, A. V. 2001, PASP, 113, 920

Leonard, D. C., Filippenko, A. V., Chornock, R., \& Weidong, L. 2002, ApJ, 124, 2506

Leonard, D. C., Li, W., Filippenko, A. V., Foley, R. J., \& Chornock, R. 2005, ApJ, 632, 450
Lisewski, A. M., Hillebrandt, W., Woosley, S. E., Niemeyer, J. C., \& Kerstein, A. R. 2000, ApJ, 537, 405L

Maund, J. R., Wheeler, J. C., Patat, F., et al. 2007, MNRAS, 381, 201

Mazzali, P. A., Benetti, S., Stehle, M., et al. 2005a, MNRAS, 357, 200

Mazzali, P. A., Benetti, S., Altavilla, G., et al. 2005b, ApJ, 623, L37

Mazzali, P. A., Röpke, F. K., Benetti, S., \& Hillebrandt, W. 2007, Science, 315, 825

Nugent, P., Phillips, M., Baron, E., Branch, D., \& Hauschildt, P. 1995, ApJ, 455, L147

Patat, F., Benetti, S., Cappellaro, E., et al. 1996, MNRAS, 278, 111

Patat, F. 2005, MNRAS, 357, 161

Patat, F., \& Romaniello, M. 2006, PASP, 118, 146

Patat, F., Chandra, P., Chevalier, R., et al. 2007, Science, 317, 924

Patat, F., Maund, J. R., Botticella, M.-T., et al. 2009, A\&A, submitted [arXiv: 0908.0942]

Perlmutter, S., Aldering, G., Goldhaber, G., et al. 1999, ApJ, 517, 565

Quimby, R., Brown, P., \& Gerardy, C. 2006a, CBET, 421

Quimby, R., Höflich, P., Kannappan, S. J., et al. 2006b, ApJ, 636, 400

Rand, R. J. 1995, ApJ, 109, 2444

Riess, A. G., Filippenko, A. V., Challis, P., et al. 1998, AJ, 116, 1009

Scarrot, S. M., Ward-Thompson, D., \& Warren-Smith, R. F. 1987, MNRAS, 224, 299

Schlegel, D. J., Finkbeiner, D. P., \& Davis, M. 1998, ApJ, 500, 525

Serkowski, K., Matheson, D. S., \& Ford, V. L. 1975, ApJ, 196, 261

Stockdale, C. J., Kelley, M., Sramek, R.A., et al. 2006, CBET, 396

Jehin, E., O'Brien, K., \& Szeifert, T. 2005, FORS1+2 User's Manual, VLT-MAN-ESO-13100-1543, Issue 78

Suzuki, S., \& Migliardi, M. 2006, IAU Circ., 8667

Tanaka, M., Mazzali, P. A., Maeda, K., \& Nomoto, K. 2006, ApJ, 645, 470

Wang, L. 2005, ApJ, 635, L33

Wang, L., \& Wheeler, J. C. 2008, Ann. Rev. Astr. Ap., 46, 433

Wang, L., Wheeler, J. C., Li, Z., \& Clocchiatti, A. 1996a, ApJ, 467, 435

Wang, L., \& Wheeler, J. C. 1996b, ApJ, 462, L27

Wang, L., Wheeler, J. C., \& Höflich, P. 1997, ApJ, 476, L27

Wang, L., Howell, D. A., Höflich, P., \& Wheeler, J. C. 2001, ApJ, 550, 1030

Wang, L., Baade, D., Höflich, P., et al. 2003, ApJ, 591, 1110

Wang, L., Baade, D., Patat, F., \& Wheeler, J. C. 2006a, CBET, 396

Wang, L., Baade, D., Höflich, P., et al. 2006b, ApJ, 653, 490

Wang, L., Baade, D., \& Patat, F. 2007, Science, 315, 212

Wang, X., Li, W., Filippenko, A. V., et al. 2008a, ApJ, 675, 626

Wang, X., Li, W., Filippenko, A.V., et al. 2008b, ApJ, 677, 1060

Whittet, D. C. B. 1992, Dust in the Galactic Environment, 2nd edn. (Bristol and Philadelphia: Institute of Physics Publishing)

Whittet, D. C. B., Martin, P. G., Hough, J. H., et al. 1992, ApJ, 386, 562 


\section{Appendix A: Additional figures}

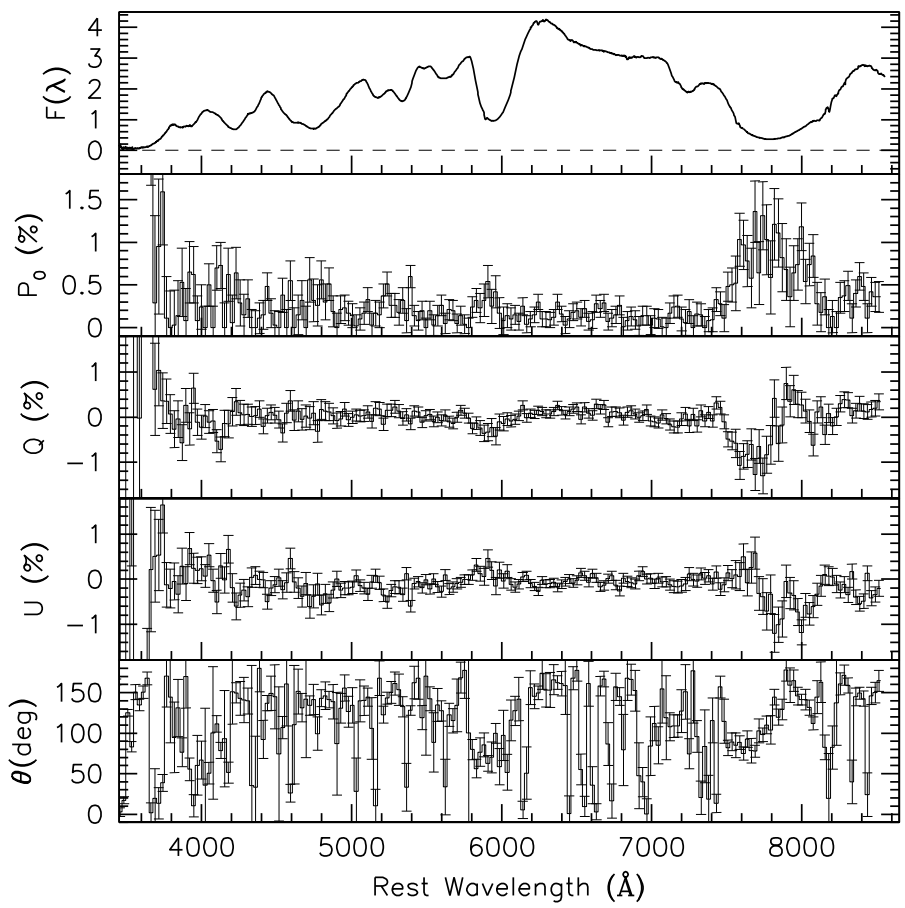

Fig. A.1. Spectropolarimetric data for SN 2006X on day -10. From top to bottom: total flux spectrum (in $10^{-15} \mathrm{erg} \mathrm{s}^{-1} \mathrm{~cm}^{-2} \AA^{-1}$ ), ISP corrected polarization degree, the Stokes parameters $Q$ and $U$ and polarization position angle on the plane of the sky.

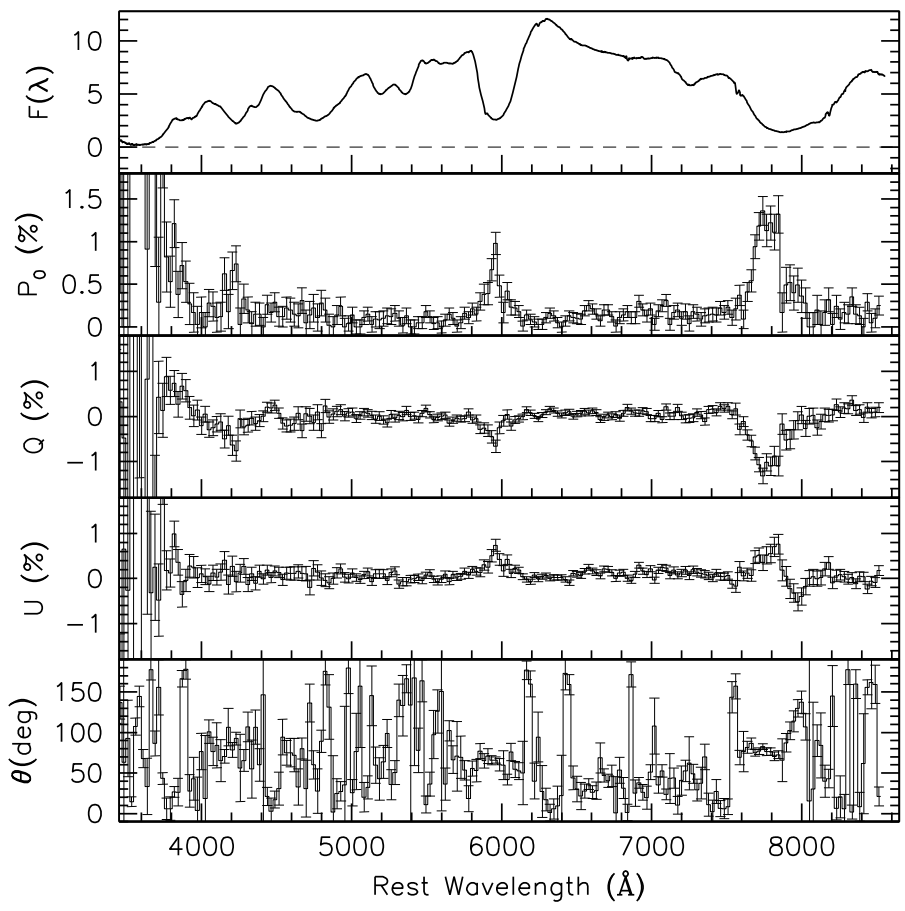

Fig. A.2. Same as Fig. A.1 for day -8 . 
F. Patat et al.: VLT spectropolarimetry of the type Ia SN 2006X, Online Material $p 2$

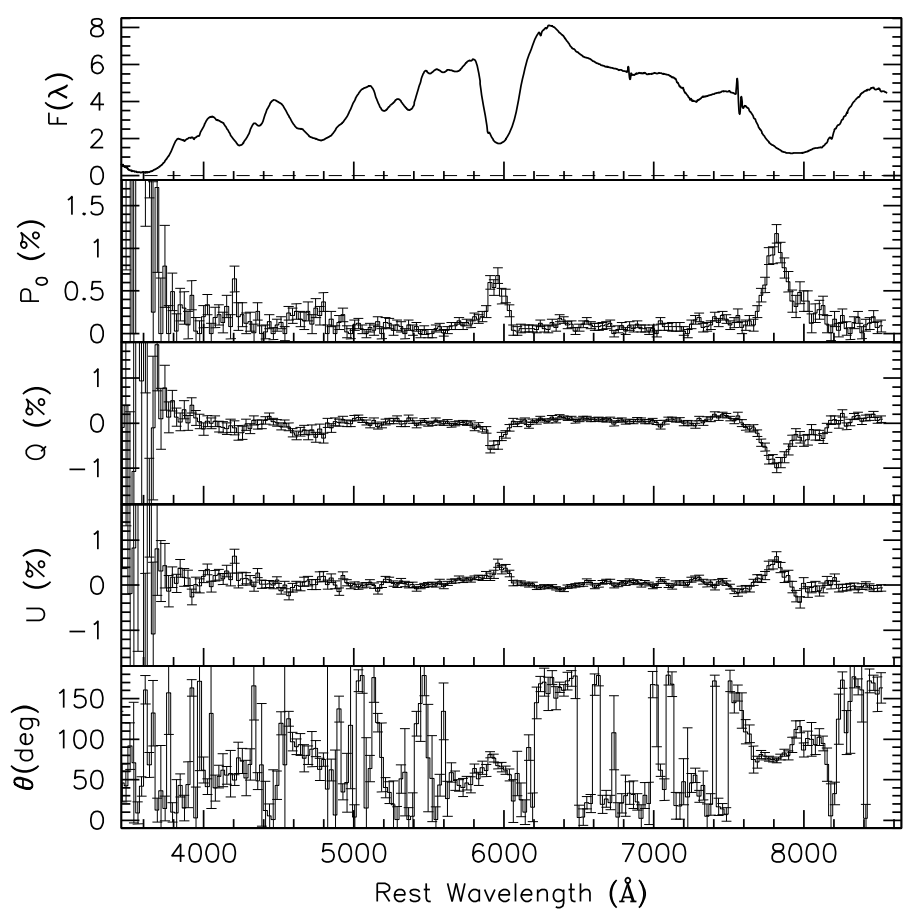

Fig. A.3. Same as Fig. A.1 for day -7 .

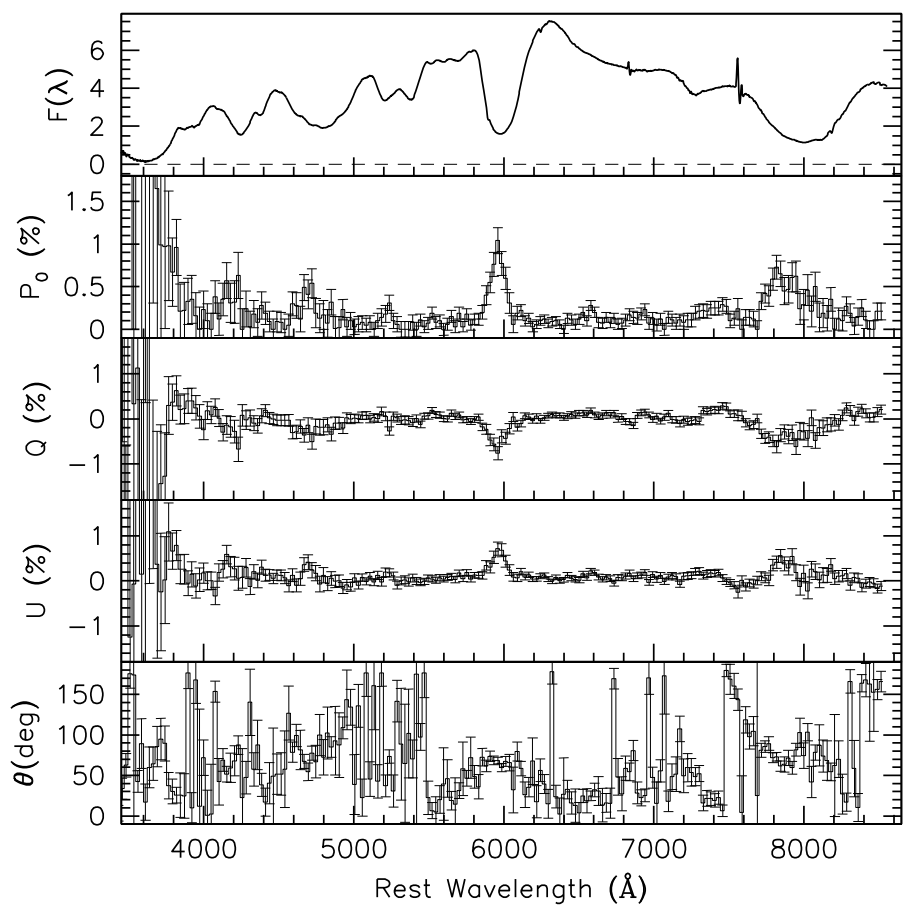

Fig. A.4. Same as Fig. A.1 for day -6 . 
F. Patat et al.: VLT spectropolarimetry of the type Ia SN 2006X, Online Material p 3

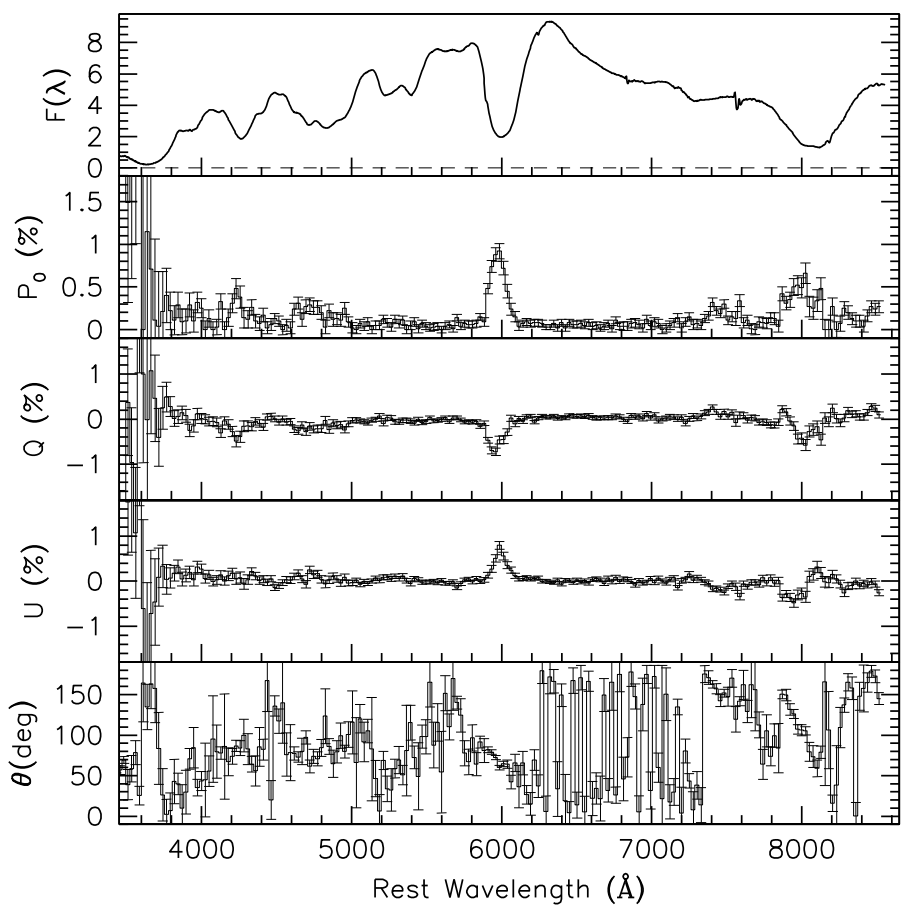

Fig. A.5. Same as Fig. A.1 for day -3 .

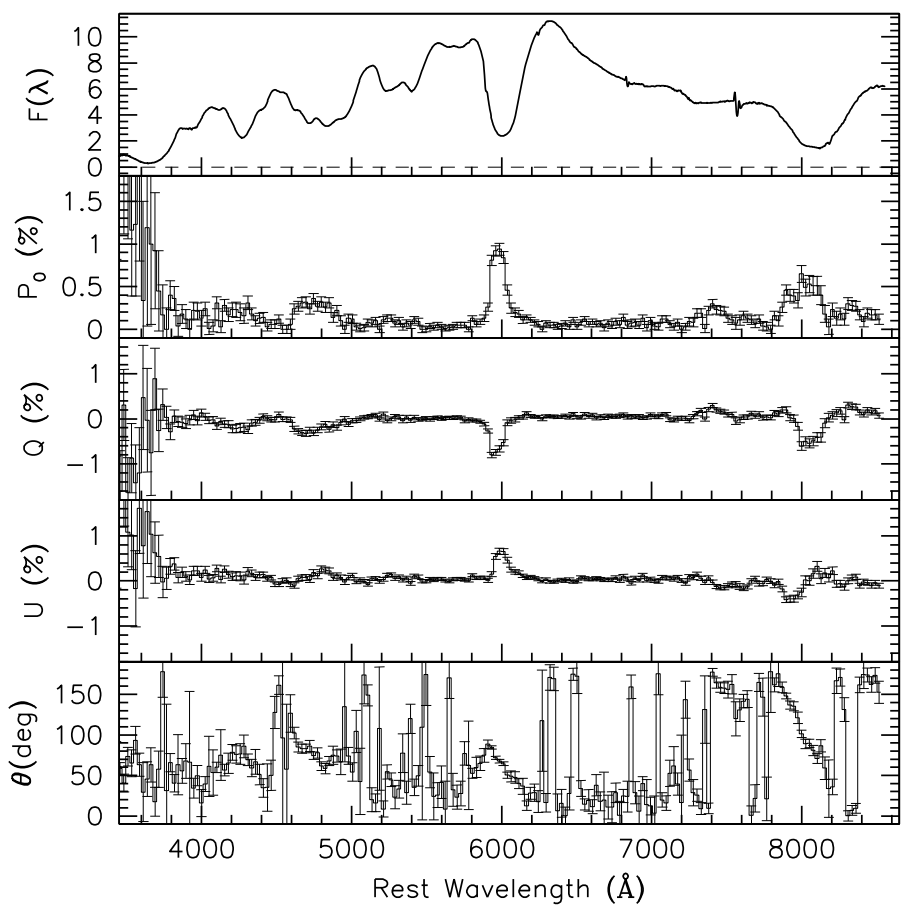

Fig. A.6. Same as Fig. A.1 for day -2 . 
F. Patat et al.: VLT spectropolarimetry of the type Ia SN 2006X, Online Material p 4

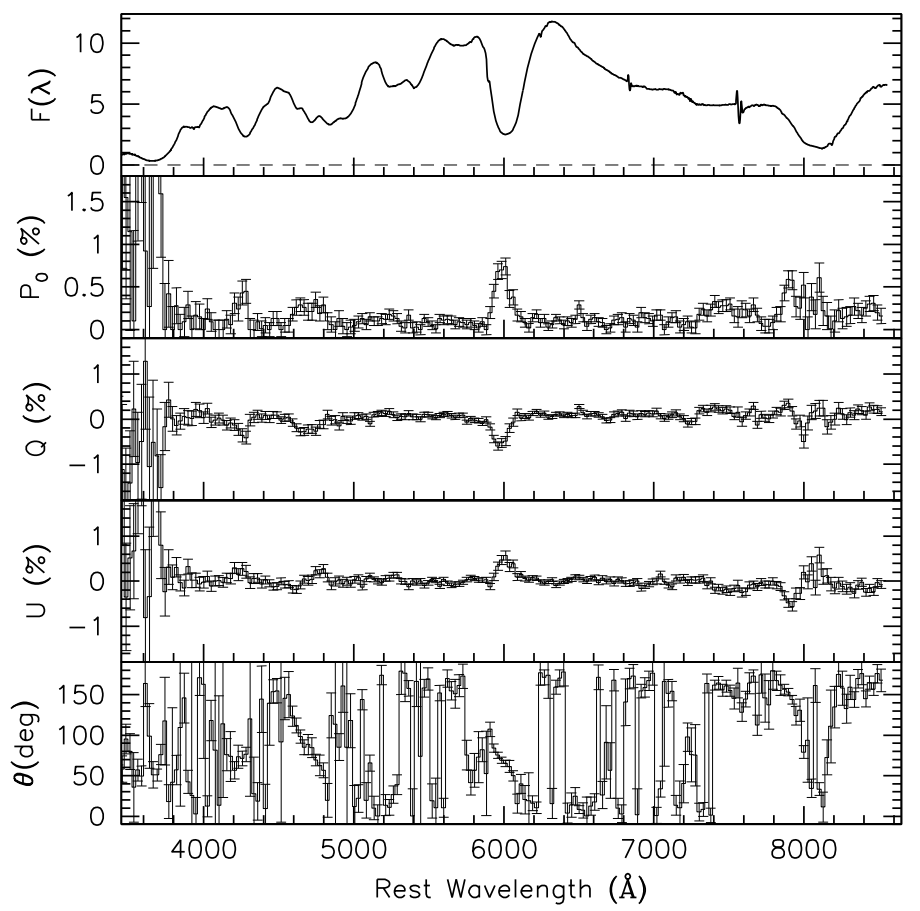

Fig. A.7. Same as Fig. A.1 for day -1 .

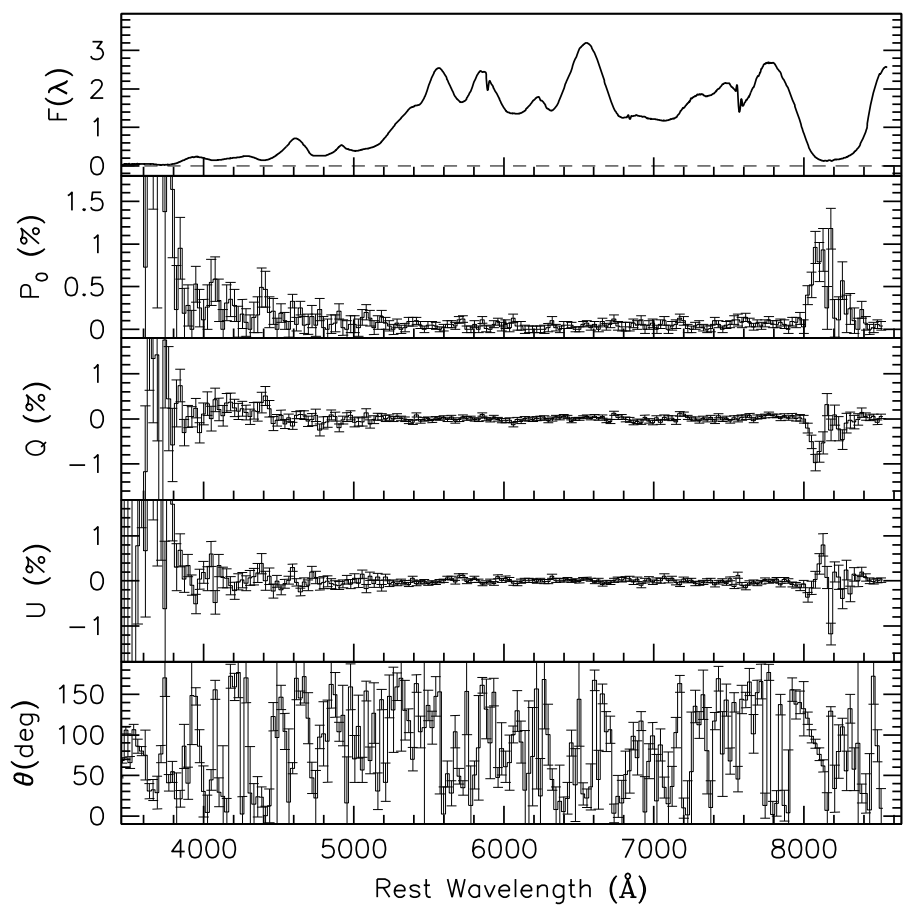

Fig. A.8. Same as Fig. A.1 for day +39 . 


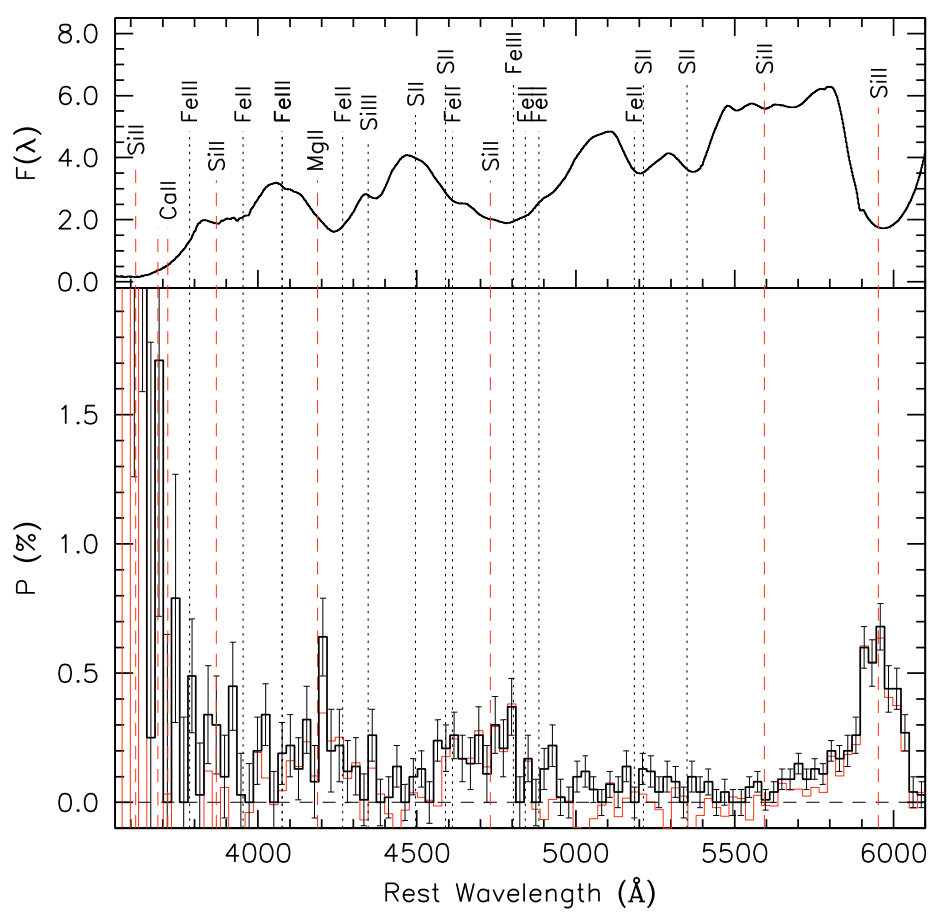

Fig. A.9. Spectropolarimetry of SN 2006X on day -7. Main line identifications are given in the upper panel. The red histogram traces the polarization along the dominant axys. Vertical dashed lines correspond to an expansion velocity of $19,000 \mathrm{~km} \mathrm{~s}^{-1}$, while the dotted lines correspond to $14,000 \mathrm{~km} \mathrm{~s}^{-1}$.

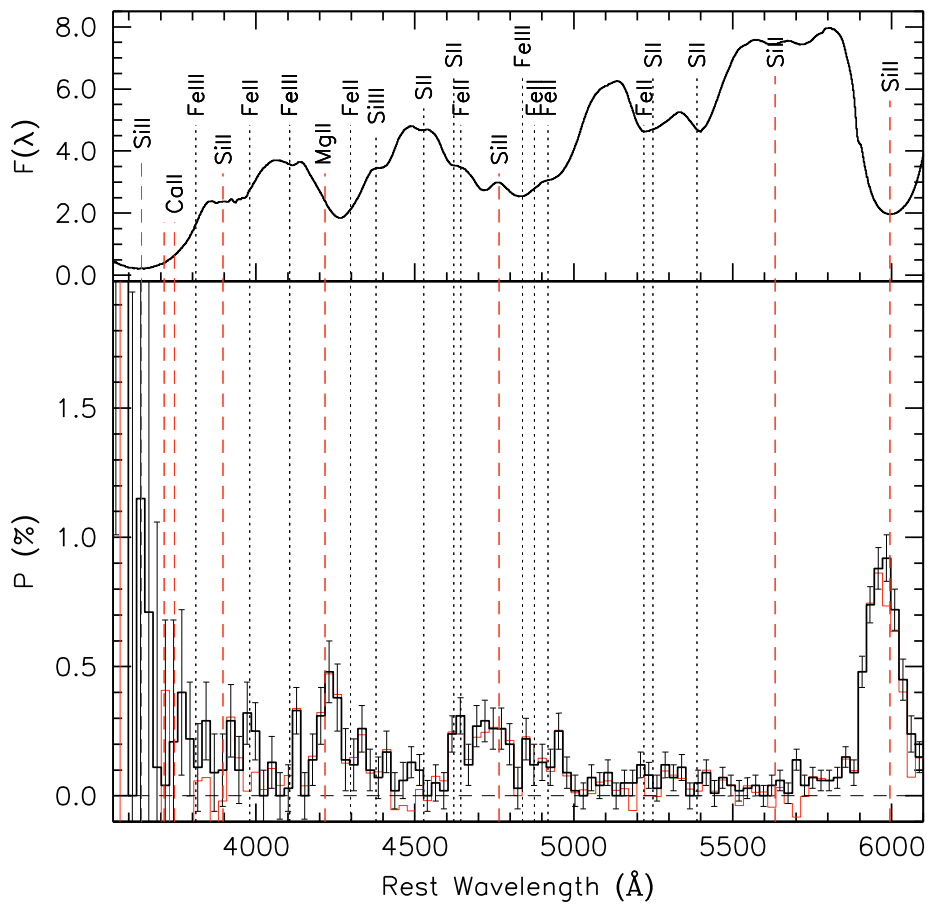

Fig. A.10. Same as Fig. A.9 for day -3. Vertical dashed lines correspond to a expansion velocity of $17,000 \mathrm{~km} \mathrm{~s}^{-1}$, while the dotted lines correspond to $12,000 \mathrm{~km} \mathrm{~s}^{-1}$. 
F. Patat et al.: VLT spectropolarimetry of the type Ia SN 2006X, Online Material p 6

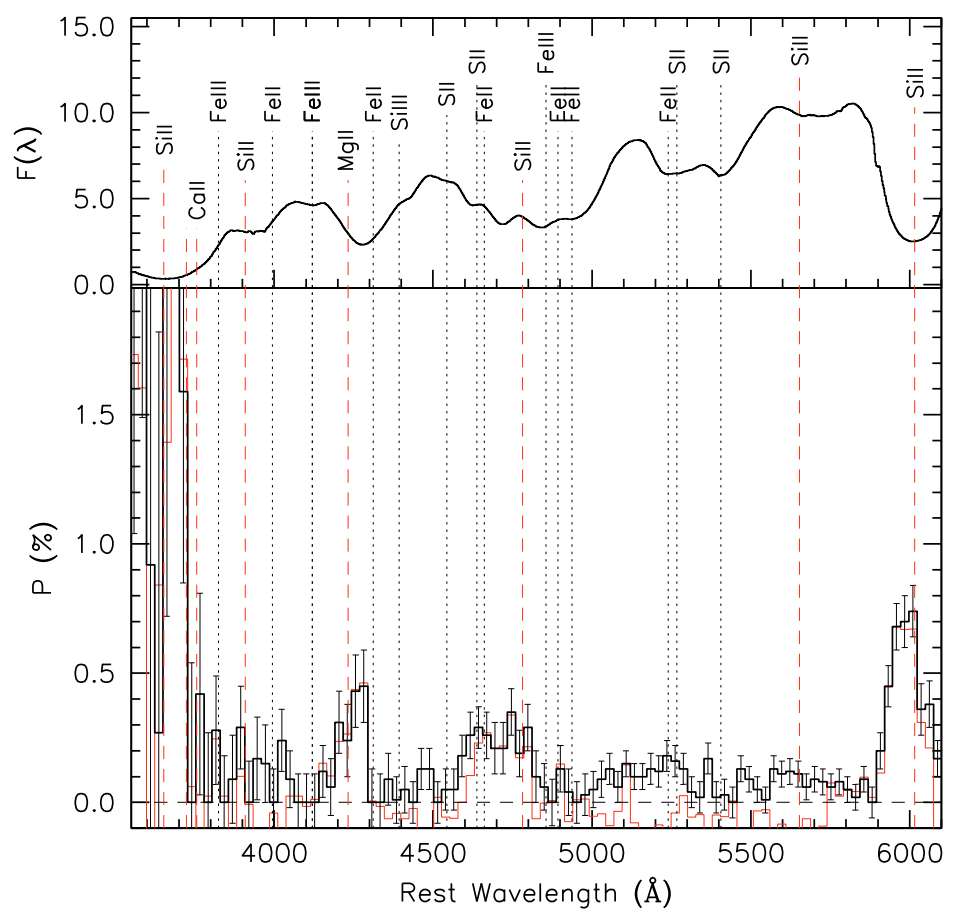

Fig. A.11. Same as Fig. A.9 for day -1 . Vertical dashed lines correspond to an expansion velocity of $16,000 \mathrm{~km} \mathrm{~s}^{-1}$, while the dotted lines correspond to $11,000 \mathrm{~km} \mathrm{~s}^{-1}$.

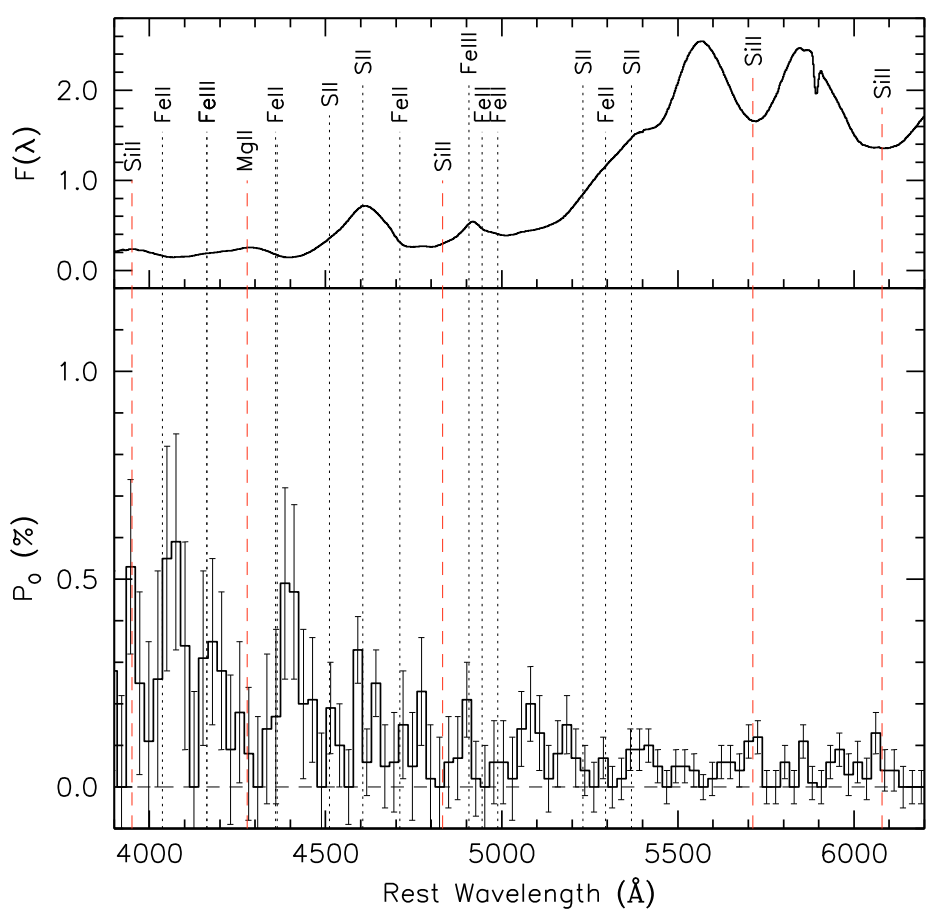

Fig. A.12. Same as Fig. A.9 for day +39. Vertical dotted lines correspond to a expansion velocity of $13,000 \mathrm{~km} \mathrm{~s}^{-1}$. 